\title{
ADAPTIVE SYMMETRIC INTERIOR PENALTY GALERKIN METHOD FOR BOUNDARY CONTROL PROBLEMS*
}

\author{
PETER BENNER ${ }^{\dagger}$ AND HAMDULLAH YÜCEL ${ }^{\ddagger}$
}

\begin{abstract}
We investigate an a posteriori error analysis of adaptive finite element approximations of linear-quadratic boundary optimal control problems under bilateral box constraints, which act on a Neumann boundary control. We use a symmetric interior Galerkin method as discretization technique. An efficient and reliable residual-type error estimator is introduced by invoking data oscillations. We then derive local upper and lower a posteriori error estimates for the boundary control problem. Adaptive mesh refinement indicated by a posteriori error estimates is applied. Numerical results are presented to illustrate the performance of the adaptive finite element approximation.
\end{abstract}

Key words. a posteriori error analysis, optimal boundary control problems, control constraints, adaptive finite element methods, discontinuous Galerkin methods

AMS subject classifications. 65N30, 65N50, 49J20, 65K10

DOI. $10.1137 / 15 \mathrm{M} 1034507$

1. Introduction. Many real-life applications such as the shape optimization of technological devices [45], the identification of parameters in environmental processes, and flow control problems $[15,18,47]$ lead to optimization problems governed by partial differential equations (PDEs). The complexity of such problems requires special care in order to obtain efficient numerical approximations for the optimization problem. One particular method is the adaptive finite element method, which consists of successive loops of the following sequence:

$$
\text { SOLVE } \rightarrow \text { ESTIMATE } \rightarrow \text { MARK } \rightarrow \text { REFINE. }
$$

The SOLVE step stands for the numerical solution of the optimization problem in a finite dimensional space defined on the given mesh. The ESTIMATE step is the key point of the adaptive finite element method. In this step, local error indicators are computed in terms of the discrete solutions without knowledge of the exact solutions. They are essential in designing algorithms for mesh adaptation, which equidistribute the computational effort and optimize the computation. Based on the information of the indicators, the MARK step selects a subset of elements subject to refinement. The refinement is then executed in the final step REFINE of the adaptive loop.

Although the adaptive finite element method, it contributed to the pioneer work of Babuška and Rheinboldt [4], has become a popular approach for the efficient solution of boundary and initial value problems for the PDEs, it has only quite recently become popular for constrained optimal control problems, initiated by Liu and Yan [43] and Becker, Kapp, and Rannacher [6]. In [43], the authors proposed a

\footnotetext{
${ }^{*}$ Received by the editors August 10, 2015; accepted for publication (in revised form) February 13, 2017; published electronically April 27, 2017.

http://www.siam.org/journals/sinum/55-2/M103450.html

Funding: The research of the second author was supported by the Forschungszentrum Dynamische Systeme (CDS): Biosystemtechnik, Otto-von-Guericke-Universität Magdeburg.

${ }^{\dagger}$ Computational Methods in Systems and Control Theory, Max Planck Institute for Dynamics of Complex Technical Systems, 39106 Magdeburg, Germany (benner@mpi-magdeburg.mpg.de).

${ }^{\ddagger}$ Corresponding author. Computational Methods in Systems and Control Theory, Max Planck Institute for Dynamics of Complex Technical Systems, 39106 Magdeburg, Germany (yuecel@ mpi-magdeburg.mpg.de). Present address: Institute of Applied Mathematics, Middle East Technical University, 06800 Ankara, Turkey (yucelh@metu.edu.tr).
} 
residual-type a posteriori error estimator for optimal control problems, while in [6] a dual-weighted goal-oriented adaptivity was proposed. We would like to refer to $[26,29,30,32,39,51,52,54,55]$ for residual-type estimators, $[8,23,25,50]$ for the dual-weighted goal-oriented approach, and the references therein for more details of recent advances. Further, in order to guarantee the success of the a posteriori error estimator theoretically, some attempts have been made in $[7,20,21,37]$ to prove the convergence of the adaptive finite element method for optimal control problems.

Adaptive mesh refinement is particularly attractive for the solution of optimal control problems, which exhibit layers or singularities in certain regions of the mesh. In this case, adaptivity allows local mesh refinement around the layers as needed, thereby achieving a desired residual bound with as few degrees of freedom as possible. The vast majority of the literature about the a posteriori error analysis of optimal control problems is for distributed optimal control problems; see, e.g., [6, 25, 26, 29, $32,39,51,52,54,50,55]$. However, there exists limited work on the numerical solution of boundary optimal control problems. The residual-type error estimators are studied in $[19,30,36,42,44]$, whereas the hierarchical-type estimators are studied in [36]. They all use continuous finite element discretizations, except for [36]. In [36], Kohls, Rösch, and Siebert use discontinuous finite elements to discretize the control. The results in [38] show that discontinuous Galerkin methods enjoy a better convergence behavior for optimal control problems exhibiting boundary layers. Optimal convergence orders are obtained if the error is computed away from boundary or interior layers. In addition, when discontinuous finite elements are used for the dicretization of the control on the Neumann boundary, the computation of the projection operator is more efficient due to the elementwise computation as described in [36, section 4.1]. Discontinuous Galerkin methods have several advantages over other types of finite element methods. For example, the state and test spaces are very easy to construct; they can naturally handle inhomogeneous boundary conditions and curved boundaries; and they have flexibility in handling nonmatching grids and in designing $h p$-adaptive grid refinement. Though these methods have been known since the 1970s, much attention has been paid only in the past few years due to the availability of cheap computing resources. We would like to refer to [3, 24, 31, 35, 33, 48] for details about discontinuous Galerkin methods. Discontinuous Galerkin methods have been studied in $[38,52,53,54,55]$ for distributed optimal control problems. To the best of our knowledge, there exists no work for boundary optimal control problems with discontinuous Galerkin discretization.

In this paper, we derive reliable and efficient a posteriori error estimators for boundary optimal control problems governed by elliptic equations, discretized by the symmetric interior penalty Galerkin (SIPG) method. We choose the SIPG as a discontinuous Galerkin method due to its symmetric property. This implies that discretization and optimization commute; see, e.g., [53]. The a posteriori error analysis of the boundary control problem includes the error in state, adjoint, control, and cocontrol and also takes data oscillations into account, in order to consider the data of the problem (coefficients of the equations, right-hand side, boundary conditions) in the most general setting as possible. We note that data oscillations are also taken into consideration in $[2,46]$ for single state equations, and in $[30,32]$ for optimal control problems.

The remainder of the paper is organized as follows: In the next section, we introduce the Neumann boundary optimal control problem governed by a second order elliptic PDE with bilateral constraints on the control. The optimality conditions are given in terms of the state, the adjoint, the control, and the cocontrol corresponding 
to the Lagrangian multiplier for the control. Section 3 describes the SIPG discretization of the boundary optimal control problem. A posteriori error estimators are given in section 4. We use a residual-type error estimator for the global discretization errors in all variables which consists of edge and element residuals. The data oscillations are also used in the error analysis. We further derive local upper and lower a posteriori error estimates for the boundary control problem. In section 5 we present some numerical results to illustrate the performance of our adaptive mesh refinement strategy. Finally, the paper ends with some conclusions.

2. The boundary control problem. We assume $\Omega$ to be an open, bounded polygonal domain in $\mathbb{R}^{2}$ with boundary $\Gamma=\overline{\Gamma_{D}} \cup \overline{\Gamma_{N}}, \Gamma_{D} \cap \Gamma_{N}=\emptyset$. We adopt standard notation from Lebesgue and Sobolev space theory (see, e.g., [1]) and refer to $(\cdot, \cdot)_{k, S}$ and $|\cdot|_{k, S},\|\cdot\|_{k, S}, k \in \mathbb{N}, S \subseteq \Omega$, as the $H^{k}(S)$-inner product and associated seminorm and norm, respectively. In addition, $c$ or $C$ denotes a general positive constant.

We consider here the following boundary control problem governed by a linearquadratic elliptic equation with constrained controls on the part of the Neumann boundary

$$
\underset{u \in U^{a d}}{\operatorname{minimize}} \frac{1}{2}\left\|y-y^{d}\right\|_{0, \Omega}^{2}+\frac{\omega}{2}\left\|u-u^{d}\right\|_{0, \Gamma_{N}}^{2}+\int_{\Gamma_{N}} r^{N} y d s
$$

subject to

$$
\begin{aligned}
-\Delta y+\alpha y & =f & & \text { in } \Omega, \\
y & =g^{D} & & \text { on } \Gamma_{D}, \\
\frac{\partial y}{\partial n} & =u+g^{N} & & \text { on } \Gamma_{N}
\end{aligned}
$$

with control constraints on a closed convex set $U^{a d}$ given by

$$
U^{a d}:=\left\{v \in L^{2}\left(\Gamma_{N}\right): u^{a} \leq v(x) \leq u^{b} \text { a.e. } x \in \Gamma_{N}\right\},
$$

where $u^{a}, u^{b} \in L^{\infty}\left(\Gamma_{N}\right)$ with $u^{a} \leq u^{b}$ for almost all $x \in \Gamma_{N}$. The function $u_{d}$, called desired control, is a guideline for the control; see, e.g., [14, 26]. Note that this formulation also allows for the special and most common case, $u_{d}=0$, i.e., there is no a priori information on the optimal control. Further, an extra coefficient $r^{N}$ is added to the standard cost functional to ensure the boundary conditions of any given adjoint function (2.7). We would like to refer to $[12,28,36]$ and references therein for similar cost functionals.

We make the following assumptions on the functions and parameters in the optimal control problem (2.1)-(2.3) to show its well-posedness:

$$
f, y^{d} \in L^{2}(\Omega), g^{D} \in H^{1 / 2}\left(\Gamma_{D}\right), u^{d}, g^{N}, r^{N} \in L^{2}\left(\Gamma_{N}\right), \omega \in \mathbb{R}_{+}, \alpha \in L^{\infty}(\Omega),
$$

and there is a constant $\alpha_{0}>0$ satisfying

$$
\alpha(x) \geq \alpha_{0} \geq 0 \quad \text { a.e. in } \Omega .
$$

Let us first consider the weak formulation of the state equation (2.2). If we define the spaces of state and test functions by

$$
Y=\left\{y \in H^{1}(\Omega):\left.y\right|_{\Gamma_{D}}=g^{D}\right\}, \quad V=\left\{v \in H^{1}(\Omega):\left.v\right|_{\Gamma_{D}}=0\right\},
$$


and the bilinear form by

$$
a(y, v)=\int_{\Omega}(\nabla y \cdot \nabla v+\alpha y v) d x,
$$

then the weak form of the state equation (2.2) for a fixed $u$ reads as follows: Find $y \in Y$ such that

$$
a(y, v)=(f, v)_{0, \Omega}+\left(u+g^{N}, v\right)_{0, \Gamma_{N}} \quad \forall v \in V .
$$

It is well known that under the above assumptions (2.4), the boundary control problem (2.1)-(2.3) admits a unique solution $(y, u) \in Y \times U^{a d}$; see, e.g., [17, 40, 42]. The solution $(y, u)$ is characterized by the existence of an adjoint $p \in V$ such that

$$
\begin{array}{ll}
a(y, v)=(f, v)_{0, \Omega}+\left(u+g^{N}, v\right)_{0, \Gamma_{N}} & \forall v \in V, \\
a(\psi, p)=-\left(y-y^{d}, \psi\right)_{0, \Omega}+\left(r^{N}, v\right)_{0, \Gamma_{N}} & \forall \psi \in V, \\
\left(\omega\left(u-u^{d}\right)-p, v-u\right)_{0, \Gamma_{N}} \geq 0 & \forall v \in U^{a d},
\end{array}
$$

where the adjoint $p$ is the solution of

$$
\begin{aligned}
-\Delta p+\alpha p & =-\left(y-y^{d}\right) & & \text { in } \Omega, \\
p & =0 & & \text { on } \Gamma_{D}, \\
\frac{\partial p}{\partial n} & =r^{N} & & \text { on } \Gamma_{N} .
\end{aligned}
$$

By invoking a Lagrange multiplier $\sigma \in L^{2}\left(\Gamma_{N}\right)$ associated with the control constraints, the optimality system (2.6) can be expressed as follows:

$$
\begin{array}{lc}
a(y, v)=(f, v)_{0, \Omega}+\left(u+g^{N}, v\right)_{0, \Gamma_{N}} & \forall v \in V, \\
a(\psi, p)=-\left(y-y^{d}, \psi\right)_{0, \Omega}+\left(r^{N}, v\right)_{0, \Gamma_{N}} & \forall \psi \in V, \\
\omega\left(u-u^{d}\right)-p+\sigma=0 & \text { a.e. in } \Gamma_{N}, \\
\sigma-\max \left\{0, \sigma+\gamma\left(u-u^{b}\right)\right\}+\min \left\{0, \sigma-\gamma\left(u^{a}-u\right)\right\}=0 & \text { a.e. in } \Gamma_{N}
\end{array}
$$

for any $\gamma>0$. Note that the equality (2.8d) is equivalent to the following pointwise complementarity system with $\sigma=\sigma^{b}-\sigma^{a}$ :

$$
\begin{array}{lll}
\sigma^{b} \geq 0, & u-u^{b} \leq 0, & \sigma^{b}\left(u-u^{b}\right)=0, \\
\sigma^{a} \geq 0, & u^{a}-u \leq 0, & \sigma^{a}\left(u^{a}-u\right)=0 .
\end{array}
$$

It is well known that (2.8) enjoys the Newton differentiability property [27], at least for $\gamma=\omega$. Therefore, we can apply a generalized (semismooth) Newton iteration. However, the infinite-dimensional generalized differentiability concept of the max- and min-functions requires a norm gap. In case of boundary controls, it is guaranteed by applying a smooth mapping as done in [22, Remark 4.3]. Due to the structure of the nonsmooth part (2.8d) the Newton iteration can be expressed in terms of an active set strategy. For any Newton iteration step, the active sets are then determined by

$$
\begin{aligned}
& \mathcal{A}_{a}=\left\{x \in \Gamma_{N}: \sigma-\gamma\left(u^{a}-u\right)<0\right\}, \\
& \mathcal{A}_{b}=\left\{x \in \Gamma_{N}: \sigma+\gamma\left(u-u^{b}\right)>0\right\},
\end{aligned}
$$

Copyright (c) by SIAM. Unauthorized reproduction of this article is prohibited. 
and the inactive set is $\mathcal{I}=\Gamma_{N} \backslash\left\{\mathcal{A}_{a} \cup \mathcal{A}_{b}\right\}$. Then, the complementarity conditions in (2.9) can be rewritten as

$$
\begin{aligned}
& u=u^{a}, \quad \sigma^{b}=0, \quad \sigma \leq 0 \quad \text { a.e. on } \mathcal{A}_{a}, \\
& u=u^{b}, \quad \sigma^{a}=0, \quad \sigma \geq 0 \quad \text { a.e. on } \mathcal{A}_{b}, \\
& u^{a}<u<u^{b}, \quad \sigma^{a}=\sigma^{b}=0, \quad \sigma=0 \quad \text { a.e. on } \mathcal{I} .
\end{aligned}
$$

3. SIPG method. We discretize our optimal control problem (2.1)-(2.3) using a discontinuous Galerkin method, namely, the SIPG discretization due to the symmetry property of its bilinear form, i.e., $a_{h}(y, v)=a_{h}(v, y)$; see, e.g., [3].

We assume that the domain $\Omega$ is polygonal such that the boundary is exactly represented by boundaries of triangles. We denote $\left\{\mathcal{T}_{h}\right\}_{h}$ as a family of shape-regular simplicial triangulations of $\Omega$. Each mesh $\mathcal{T}_{h}$ consists of closed triangles such that $\bar{\Omega}=\bigcup_{K \in \mathcal{T}_{h}} \bar{K}$ holds. We assume that the mesh is regular in the following sense: For different triangles $K_{i}, K_{j} \in \mathcal{T}_{h}, i \neq j$, the intersection $K_{i} \cap K_{j}$ is either empty or a vertex or an edge, i.e., hanging nodes are not allowed. The diameter of an element $K$ and the length of an edge $E$ are denoted by $h_{K}$ and $h_{E}$, respectively.

We split the set of all edges $\mathcal{E}_{h}$ into the set $\mathcal{E}_{h}^{0}$ of interior edges, the set $\mathcal{E}_{h}^{D}$ of Dirichlet boundary edges, and the set $\mathcal{E}_{h}^{N}$ of Neumann boundary edges so that $\mathcal{E}_{h}=\mathcal{E}_{h}^{0} \cup \mathcal{E}_{h}^{B}$ with $\mathcal{E}_{h}^{B}=\mathcal{E}_{h}^{D} \cup \mathcal{E}_{h}^{N}$. Let the edge $E$ be a common edge for two elements $K$ and $K^{e}$. For a piecewise continuous scalar function $y$, there are two traces of $y$ along $E$, denoted by $\left.y\right|_{E}$ from inside $K$ and $\left.y^{e}\right|_{E}$ from inside $K^{e}$. The jump and average of $y$ across the edge $E$ are defined by

$$
\left.\llbracket y \rrbracket=\left.y\right|_{E} \mathbf{n}_{K}+\left.y^{e}\right|_{E} \mathbf{n}_{K^{e}}, \quad\{y\}\right\}=\frac{1}{2}\left(\left.y\right|_{E}+\left.y^{e}\right|_{E}\right),
$$

where $\mathbf{n}_{K}$ (resp., $\mathbf{n}_{K^{e}}$ ) denotes the unit outward normal to $\partial K$ (resp., $\partial K^{e}$ ).

Similarly, for a piecewise continuous vector field $\nabla y$, the jump and average across an edge $E$ are given by

$$
\llbracket \nabla y \rrbracket=\left.\nabla y\right|_{E} \cdot \mathbf{n}_{K}+\left.\nabla y^{e}\right|_{E} \cdot \mathbf{n}_{K^{e}}, \quad\{\nabla y\}=\frac{1}{2}\left(\left.\nabla y\right|_{E}+\left.\nabla y^{e}\right|_{E}\right) .
$$

For a boundary edge $E \in K \cap \Gamma$, we set $\{\{\nabla y\}=\nabla y$ and $\llbracket y \rrbracket=y \mathbf{n}$, where $\mathbf{n}$ is the outward normal unit vector on $\Gamma$.

Recall that in discontinuous Galerkin methods, the state and test spaces consist of discontinuous polynomials. That is, no continuity constraints are explicitly imposed on the state and test functions across the element interfaces. As a consequence, weak formulations must include jump terms across interfaces, and typically penalty terms are added to control the jump terms. Then, we define the spaces of test functions, the discrete states, and controls by

$$
\begin{aligned}
V_{h}=Y_{h} & =\left\{y \in L^{2}(\Omega):\left.y\right|_{K} \in \mathbb{P}^{1}(K) \quad \forall K \in \mathcal{T}_{h}\right\}, \\
U_{h, N} & =\left\{u \in L^{2}\left(\Gamma_{N}\right):\left.u\right|_{E} \in \mathbb{P}^{1}(E) \quad \forall E \in \mathcal{E}_{h}^{N}\right\},
\end{aligned}
$$

respectively. $\mathbb{P}^{1}(K)$ (resp., $\mathbb{P}^{1}(E)$ ) is the set of linear polynomials in $K$ (resp., on $E)$. Note that the space $Y_{h}$ of discrete states and the space of test functions $V_{h}$ are identical due to the weak treatment of boundary conditions in discontinuous Galerkin methods. We then introduce the following (bi)linear forms $\forall(y, u, v) \in Y_{h} \times U_{h, N} \times V_{h}$ 
according to

$$
\begin{aligned}
a_{h}(y, v)= & \sum_{K \in \mathcal{T}_{h}} \int_{K}(\nabla y \cdot \nabla v+\alpha y v) d x \\
& -\sum_{E \in \mathcal{E}_{h}^{0} \cup \mathcal{E}_{h}^{D}} \int_{E}(\{\nabla y\} \cdot \llbracket v \rrbracket+\{\{\nabla v\} \cdot \llbracket y \rrbracket) d s \\
& +\sum_{E \in \mathcal{E}_{h}^{0} \cup \mathcal{E}_{h}^{D}} \frac{\sigma_{0}}{h_{E}} \int_{E} \llbracket y \rrbracket \cdot \llbracket v \rrbracket d s, \\
b_{h}(u, v)= & \sum_{E \in \mathcal{E}_{h}^{N}} \int_{E} u v d s, \\
l_{h}(v)= & \sum_{K \in \mathcal{T}_{h}} \int_{K} f v d x+\sum_{E \in \mathcal{E}_{h}^{D}} \int_{E} g^{D}\left(\frac{\sigma_{0}}{h_{E}} \mathbf{n}_{E} \cdot \llbracket v \rrbracket-\{\{\nabla v\}\}\right) d s \\
& +\sum_{E \in \mathcal{E}_{h}^{N}} \int_{E} g^{N} v d s,
\end{aligned}
$$

where the parameter $\sigma_{0} \in \mathbb{R}_{0}^{+}$is called the penalty parameter, which should be sufficiently large to ensure the stability of the discontinuous Galerkin discretization, independent of the mesh size $h$. However, it depends on the position of the edge $E$. As a threshold, the value of the penalty parameter $\sigma$ on the boundary edges $E \in \mathcal{E}^{B}$ is twice the one on the interior edges $E \in \mathcal{E}^{0}$ [48, section 2.7.1]. Further, large penalty parameters decrease the jumps across element interfaces, which can affect the numerical approximation. The discontinuous Galerkin approximation converges to the continuous Galerkin approximation as the penalty parameter goes to infinity (see, e.g., [11] for details).

Now, we mention some results, obtained by the (bi)linear forms (3.4). The bilinear form $a_{h}(\cdot, \cdot)$ is consistent with the state equation (2.2) for a fixed given control $u$ in the following sense: If $y$ satisfies $(2.2)$, then

$$
\begin{aligned}
(3.5) a_{h}(y, v)= & (f, v)_{0, \Omega}+\left(u+g^{N}, v\right)_{0, \Gamma_{N}}+\sum_{E \in \mathcal{E}_{h}^{D}}\left(y, \frac{\sigma_{0}}{h_{E}} \mathbf{n}_{E} \cdot \llbracket v \rrbracket-\{\{\nabla v\})_{0, E}\right. \\
& +\sum_{E \in \mathcal{E}_{h}^{N}}\left(\mathbf{n}_{E} \cdot \nabla y, v\right)_{0, E} \quad \forall v \in V_{h} .
\end{aligned}
$$

We then define the SIPG approximation $y_{h}$ of the solution $y$ of the state system (2.2) for a fixed given control $u_{h}=u$ such that

$$
a_{h}\left(y_{h}, v\right)=l_{h}(v)+b_{h}\left(u_{h}, v\right) \quad \forall v \in V_{h} .
$$

Thus, we have the following orthogonality relation:

$$
a_{h}\left(y-y_{h}, v\right)=0 \quad \forall v \in V_{h} .
$$

We need the following trace and inverse inequalities, which will be used frequently in the a posteriori error analysis (see, e.g., $[10,34])$ :

$$
\begin{array}{ll}
\|v\|_{0, \partial S} \leq c_{t r}\|v\|_{1, S} & \forall v \in H^{1}(S), \\
\|v\|_{0, \partial S} \leq c_{t r}\left(h_{S}^{-1}\|v\|_{0, S}^{2}+h_{S}\|\nabla v\|_{0, S}^{2}\right)^{1 / 2} & \forall v \in H^{1}(S),
\end{array}
$$

Copyright (c) by SIAM. Unauthorized reproduction of this article is prohibited. 
and

$$
|v|_{j, S} \leq c_{i n v} h_{S}^{i-j}|v|_{i, S} \quad \forall v \in \mathbb{P}_{k}(S), \quad 0 \leq i \leq j \leq 2,
$$

where $S$ is a bounded domain with a sufficiently smooth (or polygonal) boundary $\partial S$. Note that the constants $c_{t r}$ in (3.8) are different for both trace inequalities. To ease the notation, they are denoted by the same notation. We can now state the continuity and coercivity of the bilinear form $a_{h}(\cdot, \cdot)$ in the following lemma [35, Lemma 3.1].

Lemma 3.1. For $a_{h}(\cdot, \cdot)$ as in $(3.4 \mathrm{a})$, the following holds.

(i)

$$
\left|a_{h}(y, v)\right| \leq 2\|y\|\|\mid\| v\|\| \quad \forall y, v \in Y_{h} .
$$

(ii) There exists a positive constant $c_{a}$ such that

$$
a_{h}(v, v) \geq c_{a}\|v\|^{2} \quad \forall v \in V+V_{h}
$$

with the following mesh-dependent energy norm

$$
\begin{aligned}
\|v\|:= & \left(\sum_{K \in \mathcal{T}_{h}}\left(\|\nabla v\|_{0, K}^{2}+\alpha\|v\|_{0, K}^{2}\right)\right. \\
& +\sum_{E \in \mathcal{E}_{h}^{0} \cup \mathcal{E}_{h}^{D}}\left(h_{E} \|\left\{\langle\nabla v\}\left\|_{0, E}^{2}+\frac{\sigma_{0}}{h_{E}}\right\| \llbracket v \rrbracket \|_{0, E}^{2}\right)\right)^{1 / 2} .
\end{aligned}
$$

The proof of (i) is an application of the Cauchy-Schwarz inequality, while the proof of (ii) is obtained by applying the trace (3.8b) and inverse (3.9) inequalities.

In the a posteriori error analysis, we invoke data oscillations, since we do not assume any regularity of the data. Then, the data of the problem (coefficients of the equation, right-hand side, boundary conditions) are approximated by using the finite element ansatz functions on the underlying triangulation. Let

$$
f_{h}, y_{h}^{d}, \alpha_{h} \in V_{h}, \quad u_{h}^{d}, g_{h}^{N}, r_{h}^{N}, u_{h}^{a}, u_{h}^{b} \in U_{h, N}
$$

denote approximations to the right-hand side $f$, the desired state $y^{d}$, the reaction term $\alpha$, the desired control $u^{d}$, the Neumann boundary conditions $g^{N}, r^{N}$, the lower bound $u^{a}$, and the upper bound $u^{b}$, respectively. Similarly, the Dirichlet boundary condition is approximated by $g_{h}^{D} \in U_{h, D}=\left\{y \in L^{2}\left(\Gamma_{D}\right):\left.y\right|_{E} \in \mathbb{P}^{1}(E) \quad \forall E \in \mathcal{E}_{h}^{D}\right\}$.

Then, the SIPG discretization of the boundary control problem $(2.1)-(2.2)$ is given as follows:

$$
\begin{aligned}
& \operatorname{minimize} J\left(y_{h}, u_{h}\right):=\frac{1}{2} \sum_{K \in \mathcal{T}_{h}}\left\|y_{h}-y_{h}^{d}\right\|_{0, K}^{2} \\
& +\sum_{E \in \mathcal{E}_{h}^{N}} \frac{\omega}{2}\left\|u_{h}-u_{h}^{d}\right\|_{0, E}^{2}+\sum_{E \in \mathcal{E}_{h}^{N}} \int_{E} r_{h}^{N} y_{h} d s \\
& \text { over }\left(y_{h}, u_{h}\right) \in Y_{h} \times U_{h}^{a d} \text {, } \\
& \text { subject to } a_{h}\left(y_{h}, v_{h}\right)=l_{h}\left(v_{h}\right)+b_{h}\left(u_{h}, v_{h}\right), \quad v_{h} \in V_{h} \text {, }
\end{aligned}
$$

Copyright (c) by SIAM. Unauthorized reproduction of this article is prohibited. 
with the discrete constraint set for the boundary controls

$$
U_{h}^{a d}=\left\{u_{h} \in U_{h, N}: u_{h}^{a} \leq u_{h} \leq u_{h}^{b}\right\} .
$$

The optimality conditions of the disretized optimization problem (3.13) involve the existence of a discrete $p_{h} \in V_{h}$ such that

$$
\begin{array}{ll}
a_{h}\left(y_{h}, v_{h}\right)=l_{h}\left(v_{h}\right)+b_{h}\left(u_{h}, v_{h}\right) & \forall v_{h} \in V_{h}, \\
a_{h}\left(\psi_{h}, p_{h}\right)=-\left(y_{h}-y_{h}^{d}, \psi_{h}\right)_{0, \Omega}+\left(r_{h}^{N}, \psi_{h}\right)_{0, \Gamma_{N}} & \forall \psi_{h} \in V_{h}, \\
\left(\omega\left(u_{h}-u_{h}^{d}\right)-p_{h}, v_{h}-u_{h}\right)_{0, \Gamma_{N}} \geq 0 & \forall v_{h} \in U_{h}^{a d} .
\end{array}
$$

As in the continuous setting, the discrete optimality system (3.14c) can be rewritten by invoking the discrete cocontrol $\sigma_{h} \in U_{h, N}$ :

$$
\begin{array}{r}
\omega\left(u_{h}-u_{h}^{d}\right)-p_{h}+\sigma_{h}=0, \\
\sigma_{h}-\max \left\{0, \sigma_{h}+\gamma\left(u_{h}-u_{h}^{b}\right)\right\}+\min \left\{0, \sigma_{h}-\gamma\left(u_{h}^{a}-u_{h}\right)\right\}=0 .
\end{array}
$$

Note that the equality $(3.15 \mathrm{~b})$ is equivalent to the following discrete complementarity system with $\sigma_{h}=\sigma_{h}^{b}-\sigma_{h}^{a}$ :

$$
\begin{array}{lll}
\sigma_{h}^{b} \geq 0, & u_{h}-u_{h}^{b} \leq 0, & \sigma_{h}^{b}\left(u_{h}-u_{h}^{b}\right)=0, \\
\sigma_{h}^{a} \geq 0, & u_{h}^{a}-u_{h} \leq 0, & \sigma_{h}^{a}\left(u_{h}^{a}-u_{h}\right)=0 .
\end{array}
$$

We then define the discrete active sets as

$$
\begin{aligned}
& \mathcal{A}_{a, h}=\bigcup\left\{x \in E \mid \sigma_{h}(x)-\gamma\left(u_{h}^{a}(x)-u_{h}(x)\right)<0, \quad \forall E \in \mathcal{E}_{h}^{N}\right\}, \\
& \mathcal{A}_{b, h}=\bigcup\left\{x \in E \mid \sigma_{h}(x)+\gamma\left(u_{h}(x)-u_{h}^{b}(x)\right)>0, \quad \forall E \in \mathcal{E}_{h}^{N}\right\},
\end{aligned}
$$

and the inactive set is $\mathcal{I}_{h}=\mathcal{E}_{h}^{N} \backslash\left\{\mathcal{A}_{a, h} \cup \mathcal{A}_{b, h}\right\}$. Further, the complementarity conditions in (3.16) can be rewritten as in the continuous setting

$$
\begin{array}{lll}
u_{h}=u_{h}^{a}, \quad \sigma_{h}^{b}=0, \quad \sigma_{h} \leq 0 & \text { on } \mathcal{A}_{a, h}, \\
u_{h}=u_{h}^{b}, \quad \sigma_{h}^{a}=0, \quad \sigma_{h} \geq 0 & \text { on } \mathcal{A}_{b, h}, \\
u_{h}^{a}<u_{h}<u^{b}, \quad \sigma_{h}^{a}=\sigma_{h}^{b}=0, & \sigma_{h}=0 \quad \text { on } \mathcal{I}_{h} .
\end{array}
$$

4. The residual-type a posteriori error estimator. We here introduce a residual-type error estimator for the optimal control problem (2.1)-(2.3), consisting of easily computable element and edge residuals with respect to the SIPG approximation. The errors in the state $y$ and adjoint $p$ are measured by the energy norm $\|\cdot\|$, which is defined in (3.12), while the errors in the control $u$ and cocontrol $\sigma$ are measured by the $L^{2}$-norm on the Neumann boundary $\Gamma_{N}$.

The residual-type error estimator $\eta$ for the SIPG approximation of the boundary control problem $(2.1)-(2.3)$ is

$$
\eta=\left(\eta_{y}^{2}+\eta_{p}^{2}+\eta_{u}^{2}\right)^{1 / 2},
$$

Copyright (c) by SIAM. Unauthorized reproduction of this article is prohibited. 
where the state, the adjoint, and the control estimators are defined according to

$$
\begin{aligned}
& \eta_{y}=\left(\sum_{K \in \mathcal{T}_{h}} \eta_{y, K}^{2}+\sum_{E^{0} \in \mathcal{E}_{h}^{0}} \eta_{y, E^{0}}^{2}+\sum_{E^{D} \in \mathcal{E}_{h}^{D}} \eta_{y, E^{D}}^{2}+\sum_{E^{N} \in \mathcal{E}_{h}^{N}} \eta_{y, E^{N}}^{2}\right)^{1 / 2} \\
& \eta_{p}=\left(\sum_{K \in \mathcal{T}_{h}} \eta_{p, K}^{2}+\sum_{E^{0} \in \mathcal{E}_{h}^{0}} \eta_{p, E^{0}}^{2}+\sum_{E^{D} \in \mathcal{E}_{h}^{D}} \eta_{p, E^{D}}^{2}+\sum_{E^{N} \in \mathcal{E}_{h}^{N}} \eta_{p, E^{N}}^{2}\right)^{1 / 2}, \\
& \eta_{u}=\left(\sum_{E^{N} \in \mathcal{E}_{h}^{N}} \eta_{u, E^{N}}^{2}\right)^{1 / 2}
\end{aligned}
$$

respectively. The element residuals $\eta_{y, K}, \eta_{p, K}$ are given by

$$
\begin{array}{ll}
\eta_{y, K}=h_{K}\left\|f_{h}+\Delta y_{h}-\alpha_{h} y_{h}\right\|_{0, K}, & K \in \mathcal{T}_{h}, \\
\eta_{p, K}=h_{K}\left\|-\left(y_{h}-y_{h}^{d}\right)+\Delta p_{h}-\alpha_{h} p_{h}\right\|_{0, K}, & K \in \mathcal{T}_{h} .
\end{array}
$$

The edge residuals $\eta_{y, E^{0}}, \eta_{p, E^{0}}$ associated with the interior edges $E^{0} \in \mathcal{E}^{0}$ are

$$
\begin{aligned}
& \eta_{y, E^{0}}=h_{E^{0}}^{1 / 2}\left\|\llbracket \nabla y_{h} \rrbracket\right\|_{0, E^{0}}+\sigma_{0} h_{E^{0}}^{-1 / 2}\left\|\llbracket y_{h} \rrbracket\right\|_{0, E^{0}}, \quad E^{0} \in \mathcal{E}_{h}^{0}, \\
& \eta_{p, E^{0}}=h_{E^{0}}^{1 / 2}\left\|\llbracket \nabla p_{h} \rrbracket\right\|_{0, E^{0}}+\sigma_{0} h_{E^{0}}^{-1 / 2}\left\|\llbracket p_{h} \rrbracket\right\|_{0, E^{0}}, \quad E^{0} \in \mathcal{E}_{h}^{0},
\end{aligned}
$$

and the boundary edge residuals $\eta_{y, E^{D}}, \eta_{p, E^{D}}$ and $\eta_{y, E^{N}}, \eta_{p, E^{N}}, \eta_{u, E^{N}}$ with respect to the Dirichlet boundary edges $E^{D} \in \mathcal{E}^{D}$ and Neumann boundary edges $E^{N} \in \mathcal{E}^{N}$ are

$$
\begin{array}{ll}
\eta_{y, E^{D}}=\sigma_{0} h_{E^{D}}^{-1 / 2}\left\|g_{h}^{D}-y_{h}\right\|_{0, E^{D}} & \text { for } E^{D} \in \mathcal{E}_{h}^{D}, \\
\eta_{p, E^{D}}=\sigma_{0} h_{E^{D}}^{-1 / 2}\left\|p_{h}\right\|_{0, E^{D}} & \text { for } E^{D} \in \mathcal{E}_{h}^{D}, \\
\eta_{y, E^{N}}=h_{E^{N}}^{1 / 2}\left\|u_{h}+g_{h}^{N}-\mathbf{n}_{E^{N}} \cdot \nabla y_{h}\right\|_{0, E^{N}} & \text { for } E^{N} \in \mathcal{E}_{h}^{N}, \\
\eta_{p, E^{N}}=h_{E^{N}}^{1 / 2}\left\|r_{h}^{N}-\mathbf{n}_{E^{N}} \cdot \nabla p_{h}\right\|_{0, E^{N}} & \text { for } E^{N} \in \mathcal{E}_{h}^{N}, \\
\eta_{u, E^{N}}=h_{E^{N}}\left\|\mathbf{n}_{E^{N}} \cdot \nabla\left(\omega\left(u_{h}-u_{h}^{d}\right)-p_{h}\right)\right\|_{0, E^{N}} & \text { for } E^{N} \in \mathcal{E}_{h}^{N} .
\end{array}
$$

We further invoke data oscillations in the error analysis

$$
\theta=\left(\theta_{y}^{2}+\theta_{p}^{2}+\theta_{u}^{2}\right)^{1 / 2},
$$

where

$$
\begin{aligned}
(4.7 \mathrm{a}) \theta_{y}^{2}= & \sum_{K \in \mathcal{T}_{h}} \underbrace{h_{K}^{2}\left(\left\|f-f_{h}\right\|_{0, K}^{2}+\left\|\left(\alpha-\alpha_{h}\right) y_{h}\right\|_{0, K}^{2}\right)}_{\theta_{y, K}^{2}}+\sum_{E \in \mathcal{E}_{h}^{D}} \underbrace{h_{E}^{-1} \sigma_{0}\left\|g^{D}-g_{h}^{D}\right\|_{0, E}^{2}}_{\theta_{y, E^{D}}^{2}} \\
& +\sum_{E \in \mathcal{E}_{h}^{N}} \underbrace{h_{E}\left\|g^{N}-g_{h}^{N}\right\|_{0, E}^{2}}_{\theta_{y, E^{N}}}, \\
\text { (4.7b) } \theta_{p}^{2}= & \sum_{K \in \mathcal{T}_{h}} \underbrace{h_{K}^{2}\left(\left\|y^{d}-y_{h}^{d}\right\|_{0, K}^{2}+\left\|\left(\alpha-\alpha_{h}\right) p_{h}\right\|_{0, K}^{2}\right)}_{\theta_{p, K}^{2}}+\sum_{E \in \mathcal{E}_{h}^{N}} \underbrace{h_{E}\left\|r^{N}-r_{h}^{N}\right\|_{0, E}^{2}}_{\theta_{p, E}^{2}}, \\
\text { (4.7c) } \theta_{u}^{2}= & \sum_{E \in \mathcal{E}_{h}^{N}} \underbrace{\left(\omega\left\|u^{d}-u_{h}^{d}\right\|_{0, E}^{2}+\left\|u^{a}-u_{h}^{a}\right\|_{0, E}^{2}+\left\|u^{b}-u_{h}^{b}\right\|_{0, E}^{2}\right)}_{\theta_{u, E^{N}}} .
\end{aligned}
$$

Copyright $\odot$ by SIAM. Unauthorized reproduction of this article is prohibited. 
4.1. Reliability of the error estimator. In this section, we derive an upper bound for the discretization errors of the state, the adjoint, the control, and the cocontrol. The reliability means that up to data oscillations (4.6), the discretization errors can be bounded by the residual-type error estimator $\eta(4.1)$.

To prove our reliability result, we need the auxiliary solutions $y\left[u_{h}\right] \in Y$ and $p\left[u_{h}\right] \in V$, which solve the following system

$$
\begin{array}{ll}
a\left(y\left[u_{h}\right], v\right)=(f, v)_{0, \Omega}+\left(u_{h}+g^{N}, v\right)_{0, \Gamma_{N}} & \forall v \in V, \\
a\left(q, p\left[u_{h}\right]\right)=-\left(y_{h}-y_{h}^{d}, q\right)_{0, \Omega}+\left(r_{h}^{N}, q\right)_{0, \Gamma_{N}} & \forall q \in V .
\end{array}
$$

By (2.6) and (4.8), we obtain

$$
\begin{aligned}
& a\left(y-y\left[u_{h}\right], v\right)=\left(u-u_{h}, v\right)_{0, \Gamma_{N}}, \\
& a\left(q, p-p\left[u_{h}\right]\right)=\left(y_{h}-y, q\right)_{0, \Omega}+\left(y^{d}-y_{h}^{d}, q\right)_{0, \Omega}+\left(r^{N}-r_{h}^{N}, q\right)_{0, \Gamma_{N}} .
\end{aligned}
$$

Then, by using Lemma 3.1 with the trace inequality (3.8a), we obtain the following relations

$$
\begin{aligned}
& \left\|y-y\left[u_{h}\right]\right\| \mid \leq c_{t r} c_{0} c_{a}^{-1}\left\|u-u_{h}\right\|_{0, \Gamma_{N}}, \\
& \left\|p-p\left[u_{h}\right]\right\| \mid \leq c_{0} c_{a}^{-1}\left(\left\|y-y_{h}\right\|_{0, \Omega}+\left\|y^{d}-y_{h}^{d}\right\|_{0, \Omega}+c_{t r}\left\|r^{N}-r_{h}^{N}\right\|_{0, \Gamma_{N}}\right),
\end{aligned}
$$

where $c_{0}=\min \left(\alpha, \alpha^{-1}\right)$.

We now find a bound, up to the control estimator $\eta_{u}$ and the data oscillation $\theta_{u}$ for the discretization errors in terms of the auxiliary state $y\left[u_{h}\right]$ and the auxiliary adjoint $p\left[u_{h}\right]$.

Lemma 4.1. Let $(y, p, u)$ and $\left(y_{h}, p_{h}, u_{h}\right)$ be the solutions of (2.6) and (3.14), respectively, and let the cocontrol $\sigma$ and the discrete cocontrol $\sigma_{h}$ be defined in (2.8) and (3.15), respectively. Assume that $U_{h}^{a d} \subset U^{a d},\left.\left(\omega\left(u_{h}-u_{h}^{d}\right)-p_{h}\right)\right|_{E \in \mathcal{E}_{h}^{N}} \in H^{1}(E)$ and that there is a $v_{h} \in U_{h}^{a d}$ such that [42]

$$
\begin{aligned}
\|\left(\omega\left(u_{h}-u_{h}^{d}\right)-\right. & \left.p_{h}, v_{h}-u\right) \|_{0, \Gamma_{N}} \\
& \leq C \sum_{E \in \mathcal{E}_{h}^{N}} h_{E}\left\|\mathbf{n}_{E} \cdot \nabla\left(\omega\left(u_{h}-u_{h}^{d}\right)-p_{h}\right)\right\|_{0, E}\left\|u-u_{h}\right\|_{0, E} .
\end{aligned}
$$

Then, there exist positive constants $C_{i}, 1 \leq i \leq 5$, depending on the regularization parameter $\omega$, the coercivity constant $c_{a}$, and $\Omega$, such that

$$
\begin{aligned}
& \left\|u-u_{h}\right\|_{0, \Gamma_{N}}+\left\|\sigma-\sigma_{h}\right\|_{0, \Gamma_{N}}+\left\|y-y_{h}\right\|+\left\|p-p_{h}\right\| \\
& \quad \leq C_{1} \eta_{u}+C_{2} \theta_{u}+C_{3} \theta_{p}+C_{4}\left\|p\left[u_{h}\right]-p_{h}\right\| \mid+C_{5}\left\|y\left[u_{h}\right]-y_{h}\right\| .
\end{aligned}
$$

Proof. In view of the definition of $\|\cdot\| \|$ in (3.12), the inequalities (4.9) and the trace inequality (3.8a), we have

$$
\begin{aligned}
(4.12 \mathrm{a})\left\|y-y_{h}\right\| \leq & c_{t r} c_{0} c_{a}^{-1}\left\|u-u_{h}\right\|_{0, \Gamma_{N}}+\left\|y\left[u_{h}\right]-y_{h}\right\| \\
(4.12 \mathrm{~b})\left\|p-p_{h}\right\| \leq \leq & c_{0} c_{a}^{-1}\left(\left\|y-y_{h}\right\|_{0, \Omega}+\left\|y^{d}-y_{h}^{d}\right\|_{0, \Omega}+c_{t r}\left\|r^{N}-r_{h}^{N}\right\|_{0, \Gamma_{N}}\right) \\
& +\left\|p\left[u_{h}\right]-p_{h}\right\| \\
\leq & c_{t r} c_{0}^{3} c_{a}^{-2}\left\|u-u_{h}\right\|_{0, \Gamma_{N}}+c_{0}^{2} c_{a}^{-1}\left\|y\left[u_{h}\right]-y_{h}\right\|+c_{0} c_{a}^{-1}\left\|y^{d}-y_{h}^{d}\right\|_{0, \Omega} \\
& +c_{0} c_{a}^{-1} c_{t r}\left\|r^{N}-r_{h}^{N}\right\|_{0, \Gamma_{N}}+\left\|p\left[u_{h}\right]-p_{h}\right\| .
\end{aligned}
$$

Copyright (C) by SIAM. Unauthorized reproduction of this article is prohibited. 
By the equalities (2.8c) and (3.15a), the inequalities (4.12), and an application of the trace inequality $(3.8 \mathrm{a})$, we find

$$
\begin{aligned}
\left\|\sigma-\sigma_{h}\right\|_{0, \Gamma_{N}} \leq & \omega\left\|u_{h}-u\right\|_{0, \Gamma_{N}}+\omega\left\|u^{d}-u_{h}^{d}\right\|_{0, \Gamma_{N}}+\left\|p-p_{h}\right\|_{0, \Gamma_{N}} \\
\leq & \left(\omega+c_{t r}^{2} c_{a}^{-2} c_{0}^{4}\right)\left\|u_{h}-u\right\|_{0, \Gamma_{N}}+\omega\left\|u^{d}-u_{h}^{d}\right\|_{0, \Gamma_{N}} \\
& +c_{t r} c_{0}\left\|p\left[u_{h}\right]-p_{h}\right\|+c_{t r} c_{a}^{-1} c_{0}^{3}\left\|y\left[u_{h}\right]-y_{h}\right\| \\
& +c_{t r} c_{a}^{-1} c_{0}^{2}\left\|y^{d}-y_{h}^{d}\right\|_{0, \Omega}+c_{t r}^{2} c_{a}^{-1} c_{0}^{2}\left\|r^{N}-r_{h}^{N}\right\|_{0, \Gamma_{N}}
\end{aligned}
$$

By the optimality inequalities (2.6c) and (3.14c), we obtain

$$
\begin{aligned}
\omega\left\|u-u_{h}\right\|_{0, \Gamma_{N}}^{2}= & \left(\omega u, u-u_{h}\right)_{0, \Gamma_{N}}-\left(\omega u_{h}, u-u_{h}\right)_{0, \Gamma_{N}} \\
\leq & \left(\omega u^{d}+p, u-u_{h}\right)_{0, \Gamma_{N}}-\left(\omega u_{h}, u-u_{h}\right)_{0, \Gamma_{N}} \\
= & -\left(\omega\left(u_{h}-u_{h}^{d}\right)-p_{h}, u-u_{h}\right)_{0, \Gamma_{N}}+\left(p-p_{h}, u-u_{h}\right)_{0, \Gamma_{N}} \\
& +\omega\left(u^{d}-u_{h}^{d}, u-u_{h}\right)_{0, \Gamma_{N}} \\
\leq & \left(\omega\left(u_{h}-u_{h}^{d}\right)-p_{h}, v_{h}-u\right)_{0, \Gamma_{N}}+\left(p-p_{h}, u-u_{h}\right)_{0, \Gamma_{N}} \\
& +\omega\left(u^{d}-u_{h}^{d}, u-u_{h}\right)_{0, \Gamma_{N}} .
\end{aligned}
$$

For the first term on the right-hand side of (4.14), in view of the assumption in (4.10), and an application of Young's inequality, we obtain

$$
\begin{aligned}
& \left(\omega\left(u_{h}-u_{h}^{d}\right)-p_{h}, v_{h}-u\right)_{0, \Gamma_{N}} \\
& \leq \frac{2}{\omega} C \sum_{E \in \mathcal{E}_{h}^{N}} h_{E}^{2}\left\|\mathbf{n}_{E} \cdot \nabla\left(\omega\left(u_{h}-u_{h}^{d}\right)-p_{h}\right)\right\|_{0, E}^{2}+\frac{C \omega}{8}\left\|u-u_{h}\right\|_{0, \Gamma_{N}}^{2} .
\end{aligned}
$$

Next, we split the second term on the right-hand side of (4.14) into two parts:

$$
\left(u-u_{h}, p-p_{h}\right)_{0, \Gamma_{N}}=\underbrace{\left(u-u_{h}, p-p\left[u_{h}\right]\right)_{0, \Gamma_{N}}}_{M_{1}}+\underbrace{\left(u-u_{h}, p\left[u_{h}\right]-p_{h}\right)_{0, \Gamma_{N}}}_{M_{2}} .
$$

Then the equations (2.8) and (4.8) yield

$$
\begin{aligned}
M_{1}= & a\left(y-y\left[u_{h}\right], p\right)-a\left(y-y\left[u_{h}\right], p\left[u_{h}\right]\right) \\
= & \left(y^{d}-y_{h}^{d}, y-y\left[u_{h}\right]\right)_{0, \Omega}+\left(y_{h}-y, y-y\left[u_{h}\right]\right)_{0, \Omega}+\left(r^{N}-r_{h}^{N}, y-y\left[u_{h}\right]\right)_{0, \Gamma_{N}} \\
= & \left(y^{d}-y_{h}^{d}, y-y\left[u_{h}\right]\right)_{0, \Omega}+\left(y_{h}-y\left[u_{h}\right], y-y\left[u_{h}\right]\right)_{0, \Omega} \\
& +\underbrace{\left(y\left[u_{h}\right]-y, y-y\left[u_{h}\right]\right)_{0, \Omega}}_{\leq 0}+\left(r^{N}-r_{h}^{N}, y-y\left[u_{h}\right]\right)_{0, \Gamma_{N}} \\
\leq & \left(y^{d}-y_{h}^{d}, y-y\left[u_{h}\right]\right)_{0, \Omega}+\left(y_{h}-y\left[u_{h}\right], y-y\left[u_{h}\right]\right)_{0, \Omega} \\
& +\left(r^{N}-r_{h}^{N}, y-y\left[u_{h}\right]\right)_{0, \Gamma_{N}} .
\end{aligned}
$$

In view of the definition of $\|\cdot\|$ in (3.12), Young's inequality, the trace inequality (3.8a), and the inequality (4.9a), we obtain

$$
\begin{aligned}
\left(y^{d}-y_{h}^{d}, y-y\left[u_{h}\right]\right)_{0, \Omega} & \leq \frac{\omega c_{a}^{2} c_{t r}^{-2} c_{0}^{-4}}{8}\left\|y-y\left[u_{h}\right]\right\|_{0, \Omega}^{2}+\frac{2 c_{a}^{-2} c_{t r}^{2} c_{0}^{4}}{\omega}\left\|y^{d}-y_{h}^{d}\right\|_{0, \Omega}^{2} \\
& \leq \frac{\omega c_{a}^{2} c_{t r}^{-2} c_{0}^{-2}}{8}\left\|y-y\left[u_{h}\right]\right\|^{2}+\frac{2 c_{a}^{-2} c_{t r}^{2} c_{0}^{4}}{\omega}\left\|y^{d}-y_{h}^{d}\right\|_{0, \Omega}^{2} \\
& \leq \frac{\omega}{8}\left\|u-u_{h}\right\|_{0, \Gamma_{N}}^{2}+\frac{2 c_{a}^{-2} c_{t r}^{2} c_{0}^{4}}{\omega}\left\|y^{d}-y_{h}^{d}\right\|_{0, \Omega}^{2},
\end{aligned}
$$

Copyright $@$ by SIAM. Unauthorized reproduction of this article is prohibited. 


$$
\begin{aligned}
\left(r^{N}-r_{h}^{N}, y-y\left[u_{h}\right]\right)_{0, \Gamma_{N}} & \leq \frac{\omega c_{a}^{2} c_{t r}^{-4} c_{0}^{-4}}{8}\left\|y-y\left[u_{h}\right]\right\|_{0, \Gamma_{N}}^{2}+\frac{2 c_{a}^{-2} c_{t r}^{4} c_{0}^{4}}{\omega}\left\|r^{N}-r_{h}^{N}\right\|_{0, \Gamma_{N}}^{2} \\
& \leq \frac{\omega c_{a}^{2} c_{t r}^{-2} c_{0}^{-2}}{8}\left\|y-y\left[u_{h}\right]\right\|^{2}+\frac{2 c_{a}^{-2} c_{t r}^{4} c_{0}^{4}}{\omega}\left\|r^{N}-r_{h}^{N}\right\|_{0, \Gamma_{N}}^{2} \\
& \leq \frac{\omega}{8}\left\|u-u_{h}\right\|_{0, \Gamma_{N}}^{2}+\frac{2 c_{a}^{-2} c_{t r}^{4} c_{0}^{4}}{\omega}\left\|r^{N}-r_{h}^{N}\right\|_{0, \Gamma_{N}}^{2}, \\
\left(y_{h}-y\left[u_{h}\right], y-y\left[u_{h}\right]\right)_{0, \Omega} & \leq \frac{\omega c_{a}^{2} c_{t r}^{-2} c_{0}^{-4}}{8}\left\|y-y\left[u_{h}\right]\right\|_{0, \Omega}^{2}+\frac{2 c_{a}^{-2} c_{t r}^{2} c_{0}^{4}}{\omega}\left\|y_{h}-y\left[u_{h}\right]\right\|_{0, \Omega}^{2} \\
& \leq \frac{\omega c_{a}^{2} c_{t r}^{-2} c_{0}^{-2}}{8}\left\|y-y\left[u_{h}\right]\right\|^{2}+\frac{2 c_{a}^{-2} c_{t r}^{4} c_{0}^{6}}{\omega}\left\|y_{h}-y\left[u_{h}\right]\right\|^{2} \\
& \leq \frac{\omega}{8}\left\|u-u_{h}\right\|_{0, \Gamma_{N}}^{2}+\frac{2 c_{a}^{-2} c_{t r}^{4} c_{0}^{6}}{\omega}\left\|y_{h}-y\left[u_{h}\right]\right\| \|^{2} .
\end{aligned}
$$

For the second term on the right of (4.16), we obtain the following estimate by the trace inequality (3.8a) and the definition of $\|\cdot\| \mid$ in (3.12):

$$
\begin{aligned}
M_{2} & \leq \frac{\omega}{8}\left\|u-u_{h}\right\|_{0, \Gamma_{N}}^{2}+\frac{2}{\omega}\left\|p\left[u_{h}\right]-p_{h}\right\|_{0, \Gamma_{N}}^{2} \\
& \leq \frac{\omega}{8}\left\|u-u_{h}\right\|_{0, \Gamma_{N}}^{2}+\frac{2 c_{t r}^{2} c_{0}^{2}}{\omega}\left\|p\left[u_{h}\right]-p_{h}\right\|^{2} .
\end{aligned}
$$

Combining (4.17)-(4.21), we obtain

$$
\begin{aligned}
\left(u-u_{h}, p-p_{h}\right)_{0, \Gamma_{N}} \leq & \frac{\omega}{2}\left\|u-u_{h}\right\|_{0, \Gamma_{N}}^{2} \\
& +\frac{2 c_{a}^{-2} c_{t r}^{2} c_{0}^{4}}{\omega}\left\|y^{d}-y_{h}^{d}\right\|_{0, \Omega}^{2}+\frac{2 c_{a}^{-2} c_{t r}^{4} c_{0}^{4}}{\omega}\left\|r^{N}-r_{h}^{N}\right\|_{0, \Gamma_{N}}^{2} \\
& +\frac{2 c_{a}^{-2} c_{t r}^{2} c_{0}^{6}}{\omega}\left\|y_{h}-y\left[u_{h}\right]\right\|\left\|^{2}+\frac{2 c_{t r}^{2} c_{0}^{2}}{\omega}\right\| p\left[u_{h}\right]-p_{h} \|^{2} .
\end{aligned}
$$

The last term on the right-hand side of (4.14) can be estimated by invoking Young's inequality again, such that

$$
\omega\left(u^{d}-u_{h}^{d}, u-u_{h}\right)_{0, \Gamma_{N}} \leq \frac{\omega}{8}\left\|u-u_{h}\right\|_{0, \Gamma_{N}}^{2}+\frac{2}{\omega}\left\|\omega\left(u^{d}-u_{h}^{d}\right)\right\|_{0, \Gamma_{N}}^{2} .
$$

Then, using (4.15), (4.22), and (4.23), we end up with

$$
\begin{aligned}
& \left\|u-u_{h}\right\|_{0, \Gamma_{N}}^{2} \\
& \leq \frac{16}{(3-C) \omega^{2}}\left(\left\|\omega\left(u^{d}-u_{h}^{d}\right)\right\|_{0, \Gamma_{N}}^{2}+c_{a}^{-2} c_{t r}^{2} c_{0}^{4}\left\|y^{d}-y_{h}^{d}\right\|_{0, \Omega}^{2}\right. \\
& \left.+c_{a}^{-2} c_{t r}^{2} c_{0}^{6}\left\|y\left[u_{h}\right]-y_{h}\right\|^{2}+c_{a}^{-2} c_{t r}^{4} c_{0}^{4}\left\|r^{N}-r_{h}^{N}\right\|_{0, \Gamma_{N}}^{2}\right) \\
& \left.+c_{t r}^{2} c_{0}^{2}\left\|p\left[u_{h}\right]-p_{h}\right\|^{2}+C \sum_{E \in \mathcal{E}_{h}^{N}} h_{E}^{2}\left\|\mathbf{n}_{E} \cdot \nabla\left(\omega\left(u_{h}-u_{h}^{d}\right)-p_{h}\right)\right\|_{0, E}^{2}\right) .
\end{aligned}
$$

Finally, combining (4.12), (4.13), and (4.24), the desired result is obtained.

It follows from Lemma 4.1 that we need to find bounds on $\left\|p\left[u_{h}\right]-p_{h}\right\|$ and $\left\|y\left[u_{h}\right]-y_{h}\right\| \mid$. Now, we derive an upper bound for the errors between auxiliary solutions and discrete solutions in terms of the error estimators and data oscillations.

Copyright (c) by SIAM. Unauthorized reproduction of this article is prohibited. 
LEMma 4.2. If $\left(y\left[u_{h}\right], p\left[u_{h}\right]\right)$ and $\left(y_{h}, p_{h}\right)$ are the solutions of (4.8) and (3.14), respectively, then

$$
\begin{aligned}
& \left\|p\left[u_{h}\right]-p_{h}\right\|^{2} \leq C\left(\eta_{p}^{2}+\theta_{p}^{2}\right), \\
& \left\|y\left[u_{h}\right]-y_{h}\right\|^{2} \leq C\left(\eta_{y}^{2}+\theta_{y}^{2}\right) .
\end{aligned}
$$

Proof. Let $e_{p}=p\left[u_{h}\right]-p_{h}$ and $\psi=e_{p}-v_{h}$, with $v_{h} \in V_{h}$ the piecewise constant on $\mathcal{T}_{h}$. By using the coercivity result (3.11), and an orthogonality relation between $p\left[u_{h}\right]-p_{h}$, i.e., $a_{h}\left(v_{h}, e_{p}\right)=0$, we obtain

$$
\begin{aligned}
c_{a}\left\|e_{p}\right\|^{2} \leq & a_{h}\left(e_{p}, e_{p}\right)-a_{h}\left(v_{h}, e_{p}\right)=a_{h}\left(\psi, e_{p}\right) \\
= & \left(y_{h}^{d}-y_{h}, \psi\right)_{0, \Omega}-\left(\sum_{K \in \mathcal{T}_{h}}\left(\nabla \psi, \nabla p_{h}\right)_{0, K}+\alpha\left(\psi, p_{h}\right)_{0, K}\right) \\
& +\sum_{E \in \mathcal{E}_{h}^{0} \cup \mathcal{E}_{h}^{D}}\left[\left(\{\nabla \psi \psi\}, \llbracket p_{h} \rrbracket\right)_{0, E}+\left(\left\{\left\{\nabla p_{h}\right\}\right\}, \llbracket \psi \rrbracket\right)_{0, E}-\frac{\sigma_{0}}{h_{E}}\left(\llbracket \psi \rrbracket, \llbracket p_{h} \rrbracket\right)_{0, E}\right] \\
& +\sum_{E \in \mathcal{E}_{h}^{N}}\left(r_{h}^{N}, \psi\right) .
\end{aligned}
$$

Integrating by parts, we see that

$$
\begin{aligned}
& \sum_{K \in \mathcal{T}_{h}}\left(\nabla p_{h}, \nabla \psi\right)_{0, K} \\
& =\sum_{K \in \mathcal{T}_{h}}\left(-\Delta p_{h}, \psi\right)_{0, K}+\sum_{E \in \mathcal{E}_{h}^{0}}\left[\left(\left\{\nabla p_{h}\right\}, \llbracket \psi \rrbracket\right)_{0, E}+\left(\llbracket \nabla p_{h} \rrbracket,\{\{\psi\})_{0, E}\right]\right. \\
& \quad+\sum_{E \in \mathcal{E}_{h}^{D}}\left(\mathbf{n}_{E} \cdot \nabla p_{h}, \psi\right)_{0, E}+\sum_{E \in \mathcal{E}_{h}^{N}}\left(\mathbf{n}_{E} \cdot \nabla p_{h}, \psi\right)_{0, E} .
\end{aligned}
$$

Now, using (4.27) in (4.26) with the addition and subtraction of the given data, we obtain

$$
\begin{aligned}
c_{a}\left\|e_{p}\right\|^{2} \leq & \sum_{K \in \mathcal{T}_{h}}\left[\left(y_{h}^{d}-y_{h}+\Delta p_{h}-\alpha_{h} p_{h}, \psi\right)_{0, K}+\left(\left(\alpha_{h}-\alpha\right) p_{h}, \psi\right)_{0, K}\right] \\
& +\sum_{E \in \mathcal{E}_{h}^{D}}\left(\mathbf{n}_{E} \cdot \nabla \psi-\frac{\sigma_{0}}{h_{E}} \psi, p_{h}\right)+\sum_{E \in \mathcal{E}_{h}^{N}}\left(r_{h}^{N}-\mathbf{n}_{E} \cdot \nabla p_{h}, \psi\right)_{0, E} \\
& \left.+\sum_{E \in \mathcal{E}_{h}^{0}}\left[(\{\nabla \psi\}\}, \llbracket p_{h} \rrbracket\right)_{0, E}-\frac{\sigma_{0}}{h_{E}}\left(\llbracket \psi \rrbracket, \llbracket p_{h} \rrbracket\right)_{0, E}-\left(\llbracket \nabla p_{h} \rrbracket,\{\{\psi\}\}\right)_{0, E}\right] .
\end{aligned}
$$

For all $\zeta \in V_{h} \cap H^{1}(\Omega)$ with $\left.\zeta\right|_{\Gamma_{D}}=0$, the orthogonality relation gives us

$$
\begin{aligned}
0= & a_{h}\left(e_{p}, p_{h}-\zeta\right)=\sum_{K \in \mathcal{T}_{h}}\left[\left(\nabla e_{p}, \nabla\left(p_{h}-\zeta\right)\right)_{0, K}+\alpha\left(e_{p}, p_{h}-\zeta\right)_{0, K}\right] \\
& -\sum_{E \in \mathcal{E}_{h}^{0}}\left(\left\{\nabla\left(p_{h}-\zeta\right)\right\}, \llbracket e_{p} \rrbracket\right)_{0, E}-\sum_{E \in \mathcal{E}_{h}^{D}}\left(\mathbf{n}_{E} \cdot \nabla\left(p_{h}-\zeta\right), e_{p}\right)_{0, E} \\
& -\sum_{E \in \mathcal{E}_{h}^{0}}\left(\left\{\nabla e_{p}\right\}, \llbracket p_{h} \rrbracket\right)_{0, E}-\sum_{E \in \mathcal{E}_{h}^{D}}\left(\mathbf{n}_{E} \cdot \nabla e_{p}, p_{h}\right)_{0, E} \\
& +\sum_{E \in \mathcal{E}_{h}^{0}} \frac{\sigma_{0}}{h_{E}}\left(\llbracket e_{p} \rrbracket, \llbracket p_{h} \rrbracket\right)_{0, E}-\sum_{E \in \mathcal{E}_{h}^{D}} \frac{\sigma_{0}}{h_{E}}\left(p_{h}, p_{h}\right)_{0, E} .
\end{aligned}
$$

By using the definition of $\psi=e_{p}-v_{h}$, where $v_{h}$ is piecewise constant on $\mathcal{T}_{h}$, and 
applying (4.29) in (4.28), we obtain

$$
\begin{aligned}
c_{a}\|\| e_{p} \|^{2} \leq & \sum_{K \in \mathcal{T}_{h}}\left[\left(y_{h}^{d}-y_{h}+\Delta p_{h}-\alpha_{h} p_{h}, \psi\right)_{0, K}+\left(\left(\alpha_{h}-\alpha\right) p_{h}, \psi\right)_{0, K}\right] \\
& -\sum_{E \in \mathcal{E}_{h}^{0}}\left[\frac{\sigma_{0}}{h_{E}}\left(\llbracket p_{h} \rrbracket, \llbracket \psi \rrbracket\right)_{0, E}+\left(\llbracket \nabla p_{h} \rrbracket,\{\{\psi\})_{0, E}\right]\right. \\
& -\sum_{E \in \mathcal{E}_{h}^{D}} \frac{\sigma_{0}}{h_{E}}\left(\psi, p_{h}\right)_{0, E}+\sum_{E \in \mathcal{E}_{h}^{N}}\left(r_{h}^{N}-\mathbf{n}_{E} \cdot \nabla p_{h}, \psi\right)_{0, E} \\
& +\sum_{K \in \mathcal{T}_{h}}\left[\left(\nabla e_{p}, \nabla\left(p_{h}-\zeta\right)\right)_{0, K}+\alpha\left(e_{p}, p_{h}-\zeta\right)_{0, K}\right] \\
& -\sum_{E \in \mathcal{E}_{h}^{0}}\left\{\left\{\nabla\left(p_{h}-\zeta\right)\right\}, \llbracket e_{p} \rrbracket\right)_{0, E}-\sum_{E \in \mathcal{E}_{h}^{D}}\left(\mathbf{n}_{E} \cdot \nabla\left(p_{h}-\zeta\right), e_{p}\right)_{0, E} \\
& -\sum_{E \in \mathcal{E}_{h}^{0}} \frac{\sigma_{0}}{h_{E}}\left(\llbracket p_{h} \rrbracket, \llbracket p_{h} \rrbracket\right)_{0, E}-\sum_{E \in \mathcal{E}_{h}^{D}} \frac{\sigma_{0}}{h_{E}}\left(p_{h}, p_{h}\right)_{0, E} .
\end{aligned}
$$

We now obtain bounds for the terms on the right-hand side of (4.30). The terms containing $\psi$ are bounded by

$$
\begin{aligned}
& \frac{1}{\lambda_{1}}\left(h_{K}^{2} \sum_{K \in \mathcal{T}_{h}}\left\|y_{h}^{d}-y_{h}+\Delta p_{h}-\alpha_{h} p_{h}\right\|_{0, K}^{2}+\left\|\left(\alpha_{h}-\alpha\right) p_{h}\right\|_{0, K}^{2}\right) \\
& \quad+\frac{1}{\lambda_{2}} \sum_{E \in \mathcal{E}_{h}^{0}} h_{E}\left\|\llbracket \nabla p_{h} \rrbracket\right\|_{0, E}^{2}+\frac{1}{\lambda_{3}} \sum_{E \in \mathcal{E}_{h}^{0}} \sigma_{0} h_{E}^{-1}\left\|\llbracket p_{h} \rrbracket\right\|_{0, E}^{2} \\
& \quad+\frac{1}{\lambda_{4}} \sum_{E \in \mathcal{E}_{h}^{D}} \sigma_{0} h_{E}^{-1}\left\|p_{h}\right\|_{0, E}^{2}+\frac{1}{\lambda_{5}} \sum_{E \in \mathcal{E}_{h}^{N}} h_{E}\left\|r_{h}^{N}-\mathbf{n}_{E} \cdot \nabla p_{h}\right\|_{0, E}^{2} \\
& \quad+\lambda_{1} \sum_{K \in \mathcal{T}_{h}} h_{K}^{-2}\|\psi\|_{0, K}^{2}+\lambda_{2} \sum_{E \in \mathcal{E}_{h}^{0}} h_{E}^{-1} \|\left\{\{\psi\}\left\|_{0, E}^{2}+\lambda_{3} \sum_{E \in \mathcal{E}_{h}^{0}} \sigma_{0} h_{E}^{-1}\right\| \llbracket \psi \rrbracket \|_{0, E}^{2}\right. \\
& \quad+\lambda_{4} \sum_{E \in \mathcal{E}_{h}^{D}} \sigma_{0} h_{E}^{-1}\|\psi\|_{0, E}^{2}+\lambda_{5} \sum_{E \in \mathcal{E}_{h}^{N}} h_{E}^{-1}\|\psi\|_{0, E}^{2}
\end{aligned}
$$

for any $\lambda_{i}>0, i=1,2,3,4,5$. To estimate the terms containing $\psi$ in (4.31), we choose $v_{h}$ as the best piecewise constant approximation of $e_{p}$. Then, using an approximation result of $[5]$

$$
\|\psi\|_{0, K} \leq c h_{K}\left\|\nabla e_{p}\right\|_{0, K}, \quad K \in \mathcal{T}_{h},
$$

with the trace inequality $(3.8 \mathrm{~b})$, we obtain

$$
\begin{aligned}
\sum_{E \in \mathcal{E}_{h}^{0}} h_{E}^{-1}\left(\|\{\{\}\}\|_{0, E}^{2}+\|\llbracket \psi \rrbracket\|_{0, E}^{2}\right) & \leq c \sum_{E \in \mathcal{E}_{h}^{0}} \sum_{K ; E \in \partial K} h_{E}^{-1}\left(h_{K}^{-1}\|\psi\|_{0, K}^{2}+h_{K}\|\nabla \psi\|_{0, K}^{2}\right) \\
& \leq c \sum_{K \in \mathcal{T}_{h}}\left\|\nabla e_{p}\right\|_{0, K}^{2}, \\
\sum_{E \in \mathcal{E}_{h}^{B}} h_{E}^{-1}\|\psi\|_{0, E}^{2} & \leq c \sum_{E \in \mathcal{E}_{h}^{B}} \sum_{K ; E \in \partial K} h_{E}^{-1}\left(h_{K}^{-1}\|\psi\|_{0, K}^{2}+h_{K}\|\nabla \psi\|_{0, K}^{2}\right) \\
& \leq c \sum_{K \in \mathcal{T}_{h}}\left\|\nabla e_{p}\right\|_{0, K}^{2} .
\end{aligned}
$$

$$
\sum_{K \in \mathcal{T}_{h}} h_{K}^{-2}\|\psi\|_{0, K}^{2} \leq c \sum_{K \in \mathcal{T}_{h}}\left\|\nabla e_{p}\right\|_{0, K}^{2}
$$

Copyright $@$ by SIAM. Unauthorized reproduction of this article is prohibited. 
Note that here we use $h_{E}^{-1} h_{K} \leq 1$, which holds by the shape regularity of the mesh. The terms containing $p_{h}-\zeta$ on the right-hand side of (4.30) are also bounded by

$$
\begin{aligned}
& \lambda_{6} \sum_{K \in \mathcal{T}_{h}}\left\|\nabla e_{p}\right\|_{0, K}^{2}+\frac{1}{\lambda_{6}} \sum_{K \in \mathcal{T}_{h}}\left\|\nabla\left(p_{h}-\zeta\right)\right\|_{0, K}^{2}+\lambda_{7} \sum_{K \in \mathcal{T}_{h}} \alpha\left\|e_{p}\right\|_{0, K}^{2} \\
& +\frac{1}{\lambda_{7}} \sum_{K \in \mathcal{T}_{h}}\left\|p_{h}-\zeta\right\|_{0, K}^{2}+\sum_{E \in \mathcal{E}^{0}} h_{E}\left\|\left\{\nabla\left(p_{h}-\zeta\right)\right\}\right\|_{0, E}^{2}+\sum_{E \in \mathcal{E}^{0}} h_{E}^{-1}\left\|\llbracket p_{h} \rrbracket\right\|_{0, E}^{2} \\
& +\sum_{E \in \mathcal{E}^{D}} h_{E}\left\|\mathbf{n}_{E} \cdot \nabla\left(p_{h}-\zeta\right)\right\|_{0, E}^{2}+\sum_{E \in \mathcal{E}^{D}} h_{E}^{-1}\left\|p_{h}\right\|_{0, E}^{2} .
\end{aligned}
$$

The terms containing $\nabla\left(p_{h}-\zeta\right)$ in $(4.33)$ are bounded by $\sum_{K \in \mathcal{T}_{h}}\left\|\nabla\left(p_{h}-\zeta\right)\right\|_{0, K}^{2}$ by using the trace and inverse inequalities. Further, the latter is bounded by $\sum_{E \in \mathcal{E}^{0}} h_{E}^{-1}\left\|\llbracket p_{h} \rrbracket\right\|_{0, E}^{2}+\sum_{E \in \mathcal{E}^{D}} h_{E}^{-1}\left\|p_{h}\right\|_{0, E}^{2}$, in view of the estimate in [35, Thm. 2.1]. Likewise, the term $\sum_{K \in \mathcal{T}_{h}}\left\|p_{h}-\zeta\right\|_{0, K}^{2}$ is also bounded by $\sum_{E \in \mathcal{E}^{0}} h_{E}\left\|\llbracket p_{h} \rrbracket\right\|_{0, E}^{2}+$ $\sum_{E \in \mathcal{E}^{D}} h_{E}\left\|p_{h}\right\|_{0, E}^{2}$.

Finally, combining the bounds in (4.31) and (4.33) with $\left\|\nabla e_{p}\right\|_{0, K} \leq\left\|e_{p}\right\|$ and $\alpha\left\|e_{p}\right\|_{0, K} \leq\left\|e_{p}\right\|$ provided that $\lambda_{i}, i=1, \ldots, 7$ are sufficiently small, the desired result (4.25a) is obtained.

The proof of $(4.25 \mathrm{~b})$ is carried out in a similar way.

Combining Lemmas 4.1 and 4.2, we obtain the following reliability estimate.

Theorem 4.3. Let $(y, p, u)$ and $\left(y_{h}, p_{h}, u_{h}\right)$ be the solutions of (2.6) and (3.14), respectively, and let the cocontrol $\sigma$ and the discrete cocontrol $\sigma_{h}$ be defined in (2.8) and (3.15), respectively. Assume that all the conditions in Lemma 4.1 hold. Then,

$$
\left\|u-u_{h}\right\|_{0, \Gamma_{N}}+\left\|\sigma-\sigma_{h}\right\|_{0, \Gamma_{N}}+\left\|y-y_{h}\right\| \mid+\left\|p-p_{h}\right\| \leq C(\eta+\theta) .
$$

Remark 4.4. We note that our reliability estimate Theorem 4.3 is proven under the assumptions $U_{h}^{a d} \subset U^{a d}$ and, hence, the inequality (4.10), as done in [42, Lemma 3.1]. If $U_{h}^{a d} \subset U^{a d}$ is not true, we need to find bounds for some extra terms; see, e.g., [42, Remark 3.2].

4.2. Efficiency of the error estimator. Here we provide a lower bound, up to data oscillations, for the discretization errors in terms of the error estimator as given in (4.1). We will show that the local error estimators can be bounded from above by the local constituents of the error, associated data oscillations, and the active part of the control. We use bubble functions as done in $[35,49]$. The element bubble functions, denoted by $b_{K}$, are defined by using the barycentric coordinates $\lambda_{j}, j=1,2,3$, of the triangle $K$,

$$
b_{K}=27 \lambda_{1} \lambda_{2} \lambda_{3}
$$

On the other hand, the edge bubble functions, denoted by $b_{E}$, are defined by

$$
\left.b_{E}\right|_{K}=4 \lambda_{1} \lambda_{2},\left.\quad b_{E}\right|_{K^{e}}=4 \lambda_{1}^{e} \lambda_{2}^{e},
$$

where $\lambda_{1}, \lambda_{2}$ (or $\lambda_{1}^{e}, \lambda_{2}^{e}$ ) are the barycentric functions of the triangle $K$ (or $K^{e}$ ) on the edge $E \in K \cap K^{e}$. Further, the bubble functions satisfy the following equalities:

$$
\left\|b_{K}\right\|_{\infty, K}=\max _{K} b_{K}=1, \quad b_{K} \in H_{0}^{1}(K) \text { and }\left\|b_{E}\right\|_{\infty, E}=\max _{\omega_{E}} b_{E}=1, \quad b_{E} \in H_{0}^{1}\left(\omega_{E}\right),
$$


where $\omega_{E}$ is the union of the two elements that share $E$ as a common edge. We recall from [49] that there exist constants, depending on the shape regularity of the triangulation $\mathcal{T}_{h}$ and polynomial degree, such that

$$
\begin{aligned}
\|v\|_{0, K}^{2} & \leq c_{1}\left(v, v b_{K}\right)_{0, K}, & & K \in \mathcal{T}_{h}, \\
\left\|v b_{K}\right\|_{0, K} & \leq c_{2}\|v\|_{0, K}, & & K \in \mathcal{T}_{h}, \\
\left|v b_{K}\right|_{1, K} & \leq c_{3} h_{K}^{-1}\|v\|_{0, K}, & & K \in \mathcal{T}_{h}, \\
\|w\|_{0, E}^{2} & \leq c_{4}\left(w, w b_{E}\right)_{0, E}, & & E \in \mathcal{E}_{h}, \\
\left\|w b_{E}\right\|_{0, E} & \leq c_{5}\|w\|_{0, E}, & & E \in \mathcal{E}_{h}, \\
\left\|w b_{E}\right\|_{0, \omega_{E}} & \leq c_{6} h_{E}^{1 / 2}\|w\|_{0, E}, & & \omega_{E}=K \cup K^{e}, \quad E=K \cap K^{e}, \\
\left|w b_{E}\right|_{1, \omega_{E}} & \leq c_{7} h_{E}^{-1 / 2}\|w\|_{0, E}, & & \omega_{E}=K \cup K^{e}, E=K \cap K^{e},
\end{aligned}
$$

for any element $K \in \mathcal{T}_{h}$, edge $E \in \mathcal{E}_{h}$, and polynomials $v$ and $w$ defined on the elements and the patch $\omega_{E}$, respectively.

In the following, for a set of elements $S$, we denote by $\|\cdot\|_{S}$ the local energy norm

$$
\begin{aligned}
\|v\|_{S}=\left(\sum_{K \in S}\left(\|\nabla v\|_{0, K}^{2}+\alpha\|v\|_{0, K}^{2}\right)\right. & \\
& \left.\quad+\sum_{E \in \mathcal{E}_{h}^{0} \cup \mathcal{E}_{h}^{D} ; E \in \partial K, K \subset S}\left(h_{E}\|\{\nabla v v\}\|_{0, E}^{2}+\frac{\sigma_{0}}{h_{E}}\|\llbracket v \rrbracket\|_{0, E}^{2}\right)\right)^{1 / 2} .
\end{aligned}
$$

LEMmA 4.5. Let $(y, p, u)$ and $\left(y_{h}, p_{h}, u_{h}\right)$ be the solutions of (2.6) and (3.14), respectively, and let the error estimators $\eta_{y, K}, \eta_{p, K}$ and the data oscillations $\theta_{y, K}, \theta_{p, K}$ be given by (4.3) and (4.7), respectively. Then, we have

$$
\begin{aligned}
& \eta_{y, K}^{2} \leq C\left(\left\|y-y_{h}\right\|_{K}^{2}+\theta_{y, K}^{2}\right), \\
& \eta_{p, K}^{2} \leq C\left(\left\|p-p_{h}\right\|_{K}^{2}+\theta_{p, K}^{2}+h_{K}^{2}\left\|y-y_{h}\right\|_{0, K}^{2}\right) .
\end{aligned}
$$

Proof. We define the residual $R=f_{h}+\Delta y_{h}-\alpha_{h} y_{h}$, and set $W=h_{K}^{2} R b_{K}$, where $b_{K}$ is the bubble function defined in (4.35). By the inequality (4.38a),

$$
h_{K}^{2}\|R\|_{0, K}^{2} \leq c_{1}(R, W)_{0, K}=c_{1}\left(\left(f+\Delta y_{h}-\alpha_{h} y_{h}, W\right)_{0, K}+\left(f_{h}-f, W\right)_{0, K}\right) .
$$

Since the exact solution satisfies $\left.(f+\Delta y-\alpha y)\right|_{K}=0$, we obtain, using integration by parts and addition and substraction of the exact data, that

$$
h_{K}^{2}\|R\|_{0, K}^{2} \leq c_{1}\left(\left(\nabla\left(y-y_{h}\right), \nabla W\right)_{0, K}+\left(f_{h}-f, W\right)_{0, K}+\left(\left(\alpha-\alpha_{h}\right) y_{h}, W\right)_{0, K}\right) .
$$

Here, we also used that $\left.W\right|_{\partial K}=0$. By the inequalities (4.38b), (4.38c), and an application of Young's inequality, we obtain

$h_{K}^{2}\|R\|_{0, K}^{2} \leq C\left(\left\|\nabla\left(y-y_{h}\right)\right\|_{0, K}^{2}+h_{K}^{2}\left\|f-f_{h}\right\|_{0, K}^{2}+h_{K}^{2}\left\|\left(\alpha-\alpha_{h}\right) y_{h}\right\|_{0, K}^{2}\right)+C \delta\|R\|_{0, K}^{2}$,

which is the desired result (4.39a) for sufficiently small $\delta$. The inequality (4.39b) can be proven analogously.

Copyright $@$ by SIAM. Unauthorized reproduction of this article is prohibited. 
Lemma 4.6. Let $(y, p, u)$ and $\left(y_{h}, p_{h}, u_{h}\right)$ be the solutions of (2.6) and (3.14), respectively, and let the error estimators $\eta_{y, K}, \eta_{p, K}$ and the data oscillations $\theta_{y, K}, \theta_{p, K}$ be given by (4.3) and (4.7), respectively. In addition, let $\omega_{E}=K \cup K^{e}$ be the union of any two elements, i.e., $K, K^{e}$ with $E=K \cap K^{e}$. Then, we have

$$
\begin{aligned}
& h_{E}\left\|\llbracket \nabla y_{h} \rrbracket\right\|_{0, E}^{2} \\
& \quad \leq C\left(\left\|y-y_{h}\right\|_{\omega_{E}}^{2}+\sum_{K=K, K^{e}} \eta_{y, K}^{2}+\sum_{K=K, K^{e}} \theta_{y, K}^{2}\right), \\
& h_{E}\left\|\llbracket \nabla p_{h} \rrbracket\right\|_{0, E}^{2} \\
& \quad \leq C\left(\left\|p-p_{h}\right\|_{\omega_{E}}^{2}+\left\|y-y_{h}\right\|_{0, \omega_{E}}^{2}+\sum_{K=K, K^{e}} \eta_{p, K}^{2}+\sum_{K=K, K^{e}} \theta_{p, K}^{2}\right) .
\end{aligned}
$$

Proof. We set $W=\llbracket \nabla y_{h} \rrbracket b_{E}$, where $b_{E}$ is the bubble edge function on $\omega_{E}$ defined in (4.36). By using the inequality (4.38d) and the fact that $\llbracket \nabla y \rrbracket=0$ on the interior edges, we obtain

$$
h_{E}\left\|\llbracket \nabla y_{h} \rrbracket\right\|_{0, E}^{2} \leq c_{4} h_{E}\left(\llbracket \nabla y_{h} \rrbracket, W\right)_{0, E}=c_{4} h_{E}\left(\llbracket \nabla\left(y_{h}-y\right) \rrbracket, W\right)_{0, E} .
$$

After integration by parts over each of the two elements of $\omega_{E}=K \cup K^{e}$, we have

$$
\left(\llbracket \nabla\left(y_{h}-y\right) \rrbracket, W\right)_{0, E}=\left(\Delta\left(y_{h}-y\right), W\right)_{0, \omega_{E}}+\left(\nabla\left(y_{h}-y\right), \nabla W\right)_{0, \omega_{E}} .
$$

Using the differential equation $-\Delta y+\alpha y=f$ and approximating the data, we obtain

$$
\begin{aligned}
h_{E}\left\|\llbracket \nabla y_{h} \rrbracket\right\|_{0, E}^{2} \\
\leq c_{4} h_{E}\left(\left(f_{h}+\Delta y_{h}-\alpha_{h} y_{h}, W\right)_{0, \omega_{E}}+\left(f-f_{h}, W\right)_{0, \omega_{E}}+\left(\left(\alpha_{h}-\alpha\right) y_{h}, W\right)_{0, \omega_{E}}\right) \\
\quad+c_{4} h_{E}\left(\left(\alpha\left(y_{h}-y\right), W\right)_{0, \omega_{E}}+\left(\nabla\left(y_{h}-y\right), \nabla W\right)_{0, \omega_{E}}\right) .
\end{aligned}
$$

Then, the inequalities (4.38f) and (4.38g) yield

$$
\begin{aligned}
& h_{E}\left\|\llbracket \nabla y_{h} \rrbracket\right\|_{0, E}^{2} \\
& \leq C h_{E}^{1 / 2}\left\|\llbracket \nabla y_{h} \rrbracket\right\|_{0, E}\left(\left\|y-y_{h}\right\|_{\omega_{E}}+\left(\sum_{K=K, K^{e}} \eta_{y, K}^{2}\right)^{1 / 2}+\left(\sum_{K=K, K^{e}} \theta_{y, K}^{2}\right)^{1 / 2}\right),
\end{aligned}
$$

which gives the desired result (4.40a) after an application of Young's inequality and the shape regularity of the mesh, i.e., $h_{E} \leq \gamma h_{K}$ with $\gamma>1$. The proof of (4.40b) is carried out in a similar way.

Lemma 4.7. Let $(y, p, u)$ and $\left(y_{h}, p_{h}, u_{h}\right)$ be the solutions of (2.6) and (3.14), respectively, and let $\eta_{y, E^{N}}, \eta_{p, E^{N}}$ and the data oscillations $\theta_{y, K}, \theta_{y, E^{N}}, \theta_{p, K}$ be given by (4.5) and (4.7), respectively, for any $E^{N} \in \partial K, K \in \mathcal{T}_{h}$. Then, we have

$$
\begin{aligned}
& \eta_{y, E^{N}}^{2} \leq C\left(\left\|y-y_{h}\right\|_{K}^{2}+\eta_{y, K}^{2}+\theta_{y, K}^{2}+\theta_{y, E^{N}}^{2}+\left\|u-u_{h}\right\|_{0, E}^{2}\right), \\
& \eta_{p, E^{N}}^{2} \leq C\left(\left\|p-p_{h}\right\|_{K}^{2}+\left\|y-y_{h}\right\|_{0, K}^{2}+\eta_{p, K}^{2}+\theta_{p, K}^{2}+\theta_{p, E^{N}}^{2}\right) .
\end{aligned}
$$

Copyright $@$ by SIAM. Unauthorized reproduction of this article is prohibited. 
Proof. We set $W=\left(\mathbf{n}_{E} \cdot \nabla y_{h}-u_{h}-g_{h}^{N}\right) b_{E}$, where $b_{E}$ is the bubble edge function. By using the inequality (4.38d) and the fact that $\mathbf{n}_{E} \cdot \nabla y=u+g^{N}$ on the Neumann boundary edges, we obtain

$$
\begin{aligned}
\eta_{y, E^{N}}^{2} & =h_{E}\left\|u_{h}+g_{h}^{N}-\mathbf{n}_{E} \cdot \nabla y_{h}\right\|_{0, E}^{2} \\
& \leq c_{4} h_{E}\left(\mathbf{n}_{E} \cdot \nabla y_{h}-u_{h}-g_{h}^{N}, W\right)_{0, E} \\
& =c_{4} h_{E}\left(\left(\mathbf{n}_{E} \cdot \nabla\left(y_{h}-y\right), W\right)_{0, E}+\left(u-u_{h}, W\right)_{0, E}+\left(g^{N}-g_{h}^{N}, W\right)_{0, E}\right) .
\end{aligned}
$$

Using integration by parts on the element $K \supset E$, the differential equation $-\Delta y+$ $\alpha y=f$, and approximating the data, we obtain

$$
\begin{aligned}
\eta_{y, E^{N}}^{2} \leq & c_{4} h_{E}\left(\left(f_{h}+\Delta y_{h}-\alpha_{h} y_{h}, W\right)_{0, K}+\left(f-f_{h}, W\right)_{0, K}+\left(\left(\alpha_{h}-\alpha\right) y_{h}, W\right)_{0, K}\right) \\
& +c_{4} h_{E}\left(\left(\alpha\left(y_{h}-y\right), W\right)_{0, K}+\left(\nabla\left(y_{h}-y\right), \nabla W\right)_{0, K}\right) \\
& +c_{4} h_{E}\left(\left(u-u_{h}, W\right)_{0, E}+\left(g^{N}-g_{h}^{N}, W\right)_{0, E}\right) .
\end{aligned}
$$

Then, the inequalities $(4.38 \mathrm{e})-(4.38 \mathrm{~g})$ yield

$\eta_{y, E^{N}}^{2} \leq C h_{E}^{1 / 2}\left\|u_{h}+g_{h}^{N}-\mathbf{n}_{E} \cdot \nabla y_{h}\right\|_{0, E}\left(\left\|y-y_{h}\right\|_{K}+\eta_{y, K}+\theta_{y, K}+\theta_{y, E^{N}}+\left\|u-u_{h}\right\|_{0, E}\right)$.

Finally, by applying Young's inequality, we obtain the desired result (4.41a). The proof of $(4.41 \mathrm{~b})$ can be verified by using the same arguments.

What is now left is to bound the estimator for the discretization error in the controls.

LEMMA 4.8. Let $(y, p, u)$ and $\left(y_{h}, p_{h}, u_{h}\right)$ be the solutions of (2.6) and (3.14), respectively, and let $\eta_{u, E^{N}}$ and $\theta_{u}$ be given by (4.5) and (4.7), respectively. Assume that all the conditions in Lemma 4.1 hold. Then, we have

$$
\begin{aligned}
\eta_{u, E^{N}}^{2} \leq C( & \left\|u-u_{h}\right\|_{0, \Gamma_{N}}^{2}+\left\|p-p_{h}\right\|_{K}^{2}+\theta_{u}^{2} \\
& \left.+h_{E}\left\|\left(\mathbf{n}_{E} \cdot \nabla\left(\omega\left(u_{h}-u_{h}^{d}\right)-p_{h}\right)\right) \chi_{\mathcal{A}_{h}}\right\|_{0, E}^{2}\right)
\end{aligned}
$$

where $\mathcal{A}_{h}$ is the union of the active sets $\mathcal{A}_{a, h}$ and $\mathcal{A}_{b, h}$.

Proof. We have that $\left(\omega\left(u-u^{d}\right)-p\right) \chi_{I}=0$ from $(2.11)$. It follows from the inverse inequality (3.9) that

$$
\begin{aligned}
\eta_{u, E^{N}}^{2}= & h_{E}\left\|\mathbf{n}_{E} \cdot \nabla\left(\omega\left(u_{h}-u_{h}^{d}\right)-p_{h}\right)\right\|_{0, E}^{2} \\
\leq & C\left\|\left(\omega\left(u_{h}-u_{h}^{d}\right)-p_{h}\right) \chi_{\mathcal{I}_{h}}\right\|_{0, E}^{2}+h_{E}\left\|\left(\mathbf{n}_{E} \cdot \nabla\left(\omega\left(u_{h}-u_{h}^{d}\right)-p_{h}\right)\right) \chi_{\mathcal{A}_{h}}\right\|_{0, E}^{2} \\
= & C\left\|\left(\omega\left(u_{h}-u_{h}^{d}\right)-p_{h}-\omega\left(u-u^{d}\right)+p\right) \chi_{\mathcal{I}_{h}}\right\|_{0, E}^{2} \\
& +h_{E}\left\|\left(\mathbf{n}_{E} \cdot \nabla\left(\omega\left(u_{h}-u_{h}^{d}\right)-p_{h}\right)\right) \chi_{\mathcal{A}_{h}}\right\|_{0, E}^{2} \\
\leq & C\left(\omega\left\|u-u_{h}\right\|_{0, E}^{2}+\omega\left\|u^{d}-u_{h}^{d}\right\|_{0, E}^{2}+\left\|p-p_{h}\right\|_{0, E}^{2}\right) \\
& +h_{E}\left\|\left(\mathbf{n}_{E} \cdot \nabla\left(\omega\left(u_{h}-u_{h}^{d}\right)-p_{h}\right)\right) \chi_{\mathcal{A}_{h}}\right\|_{0, E}^{2} \cdot
\end{aligned}
$$

This is the desired inequality.

Copyright $@$ by SIAM. Unauthorized reproduction of this article is prohibited. 
Now, we derive an upper bound consisting of the local constituents of the error, associated data oscillations, and the active part of the control, for the local error estimators. We state the efficiency estimate in the following theorem.

Theorem 4.9. Let $(y, p, u)$ and $\left(y_{h}, p_{h}, u_{h}\right)$ be the solutions of (2.6) and (3.14), respectively, and the error estimator $\eta$ and data oscillation $\theta$ be given as in (4.2) and (4.6), respectively. Assume that all the conditions in Lemma 4.1 hold. Then, it holds

$$
\begin{aligned}
\eta \leq C( & \left\|u-u_{h}\right\|_{0, \Gamma_{N}}+\left\|y-y_{h}\right\|\left|+\left\|p-p_{h}\right\|\right|+\theta \\
& \left.+\sum_{E \in \mathcal{E}_{h}^{N}} h_{E}\left\|\left(\mathbf{n}_{E} \cdot \nabla\left(\omega\left(u_{h}-u_{h}^{d}\right)-p_{h}\right)\right) \chi_{\mathcal{A}_{h}}\right\|_{0, E}\right) .
\end{aligned}
$$

Proof. By the definition of the energy norm defined in (3.12), and the fact that $\llbracket y \rrbracket=0$ on the interior edges and $y=g^{D}$ on the Dirichlet boundary edges, we can easily derive

$$
\begin{aligned}
\sum_{E \in \mathcal{E}_{h}^{0}} \frac{\sigma_{0}^{2}}{h_{E}}\left\|\llbracket y_{h} \rrbracket\right\|_{0, E}^{2}+\sum_{E \in \mathcal{E}_{h}^{D}} \frac{\sigma_{0}^{2}}{h_{E}}\left\|g_{h}^{D}-y_{h}\right\|_{0, E}^{2} & \\
& \leq C\left(\left\|y-y_{h}\right\|^{2}+\sum_{E \in \mathcal{E}_{h}^{D}} h_{E}^{-1}\left\|g^{D}-g_{h}^{D}\right\|_{0, E}^{2}\right) .
\end{aligned}
$$

Analogously, we obtain

$$
\sum_{E \in \mathcal{E}_{h}^{0}} \frac{\sigma_{0}^{2}}{h_{E}}\left\|\llbracket p_{h} \rrbracket\right\|_{0, E}^{2}+\sum_{E \in \mathcal{E}_{h}^{D}} \frac{\sigma_{0}^{2}}{h_{E}}\left\|p_{h}\right\|_{0, E}^{2} \leq C\left\|p-p_{h}\right\|^{2} .
$$

Then, the combination of the results in Lemmas 4.5-4.8 with the inequalities (4.44)(4.45) gives the assertion (4.43).

Remark 4.10. In Theorems 4.3 and 4.9, we show the reliability and efficiency of our estimator $\eta$. However, the estimator $\eta_{u}$ contributed from the approximation error of the control cannot lead to a localization of refinement of the inactive set in some notable cases. In this case, this key step should be improved numerically. Therefore, in our numerical experiments, we use

$$
\widetilde{\eta}_{u}=\sum_{E \in \mathcal{E}_{h}^{N}} h_{E}\left\|\left(\mathbf{n}_{E} \cdot \nabla\left(\omega\left(u_{h}-u_{h}^{d}\right)-p_{h}\right)\right) \chi_{\mathcal{I}_{h}}\right\|_{0, E}
$$

as an indicator of the control instead of $\eta_{u}(4.2 \mathrm{c})$. The same problem is also observed for the control indicators proposed in [30, 36, 42].

In computing, we further approximate the characteristic function $\chi_{\mathcal{I}_{h}}$ by

$$
\chi_{\mathcal{I}_{h}}=\frac{\left(u_{h}-u_{h}^{a}\right)\left(u_{h}^{b}-u_{h}\right)}{h^{\mu}+\left(u_{h}-u_{h}^{a}\right)\left(u_{h}^{b}-u_{h}\right)}
$$

where $\mu>0$ as done in [41]. 


\section{Numerical experiments.}

5.1. The adaptive loop. An adaptive procedure for the SIPG discretization of the optimization problem (2.1)-(2.3) consists of successive loops of the sequence given in (1.1). The SOLVE step is the numerical solution of the optimal control problem with respect to the given triangulation $\mathcal{T}_{h}$ using the SIPG discretization. By using the primal dual active set algorithm as a semismooth Newton step (see, e.g., [9]), we solve the following discrete linear system

$$
\left(\begin{array}{cccc}
\mathcal{M} & \cdot & \mathcal{K}^{T} & \cdot \\
\cdot & \omega \mathcal{M}_{B} & -\mathcal{M} & \mathcal{M}_{B} \\
\mathcal{K} & -\mathcal{M}_{B} & \cdot & \cdot \\
\cdot & \gamma \chi_{\mathcal{A}_{h}} & \cdot & \chi_{I_{h}}
\end{array}\right)\left(\begin{array}{c}
y_{h} \\
u_{h} \\
p_{h} \\
\sigma_{h}
\end{array}\right)=\left(\begin{array}{c}
\mathcal{M} y_{h}^{d} \\
\omega \mathcal{M}_{B} u_{h}^{d} \\
\mathcal{F} \\
\gamma\left(\chi_{\mathcal{A}_{a, h}} u_{h}^{a}+\chi_{\mathcal{A}_{b, h}} u_{h}^{b}\right)
\end{array}\right),
$$

where $\mathcal{A}_{h}=\mathcal{A}_{a, h} \cup \mathcal{A}_{b, h} \cdot \chi_{\mathcal{A}_{a, h}}, \chi_{\mathcal{A}_{b, h}}$, and $\chi_{\mathcal{A}_{h}}$ denote the characteristic functions of $\mathcal{A}_{a, h}, \mathcal{A}_{b, h}$, and $\mathcal{A}_{h}$, respectively, defined on the Neumann boundary $\mathcal{E}_{h}^{N} . \mathcal{M}$ and $\mathcal{M}_{B}$ are mass matrices on the domain and boundary, respectively. $\mathcal{K}$ and $\mathcal{F}$ correspond to the bilinear form $a_{h}\left(y_{h}, v_{h}\right)$ and the linear form $l_{h}\left(v_{h}\right)$ defined in (3.4), respectively.

For the ESTIMATE step, the residual error estimators $\eta_{y}, \eta_{p}$, and $\eta_{u}$ defined in section 4 are used. In the MARK step of the adaptive loop, the edges and elements for the refinement are specified by using the a posteriori error estimator and by choosing subsets $\mathcal{M}_{K} \subset \mathcal{T}_{h}$ and $\mathcal{M}_{E} \subset \mathcal{E}_{h}$ such that the following bulk criterion [16] is satisfied for a given marking parameter $\Theta$ with $0<\Theta<1$ :

$$
\begin{aligned}
& \Theta \sum_{K \in \mathcal{T}_{h}}\left(\eta_{K}^{y}\right)^{2}+\left(\eta_{K}^{p}\right)^{2} \leq \sum_{K \in \mathcal{M}_{K}}\left(\eta_{K}^{y}\right)^{2}+\left(\eta_{K}^{p}\right)^{2}, \\
& \Theta \sum_{E \in \mathcal{E}_{h}}\left(\eta_{E}^{y}\right)^{2}+\left(\eta_{E}^{p}\right)^{2}+\left(\eta_{E}^{u}\right)^{2} \leq \sum_{E \in \mathcal{M}_{E}}\left(\eta_{E}^{y}\right)^{2}+\left(\eta_{E}^{p}\right)^{2}+\left(\eta_{E}^{u}\right)^{2} .
\end{aligned}
$$

Bigger values for the parameter $\Theta$ will result in more refinement of triangles in one loop and smaller $\Theta$ will result in a more optimal grid but more refinement loops. We note that the data oscillations may be included in the bulk criterion as done for the estimator $\eta$ in (5.2). Finally, in the REFINE step, the marked elements are refined by longest edge bisection, whereas the elements of the marked edges are refined by bisection [13]. The adaptive procedure ends after a sequence of mesh refinements up to attaining a solution with an estimated error within a prescribed tolerance.

5.2. Numerical results. We now present several numerical results in order to examine the quality of the derived estimators in section 4 and the performance of the adaptive loop introduced in section 5.1. We use piecewise linear polynomials for the approximation of the state, the adjoint, the control, and the cocontrol. The penalty parameter $\sigma_{0}$ in the SIPG is chosen as $\sigma_{0}=6$ on the interior edges and 12 on the boundary edges. The parameter $\gamma$ used in the definition of the active and inactive sets is chosen as equal to the regularization parameter $\omega$. The effectivity index is calculated according to

$$
\text { effectivity index }=\frac{\eta_{y}+\eta_{p}+\eta_{u}}{\left\|y-y_{h}\right\||+|\left\|p-p_{h}\right\| \mid+\left\|u-u_{h}\right\|_{0, \Gamma_{N}}+\left\|\sigma-\sigma_{h}\right\|_{0, \Gamma_{N}}} .
$$

We finally define the projection of the control such that

$$
\operatorname{Proj}_{u^{a}, u^{b}}(v)=\max \left\{u^{a}, \min \left\{u^{b}, v\right\}\right\} .
$$




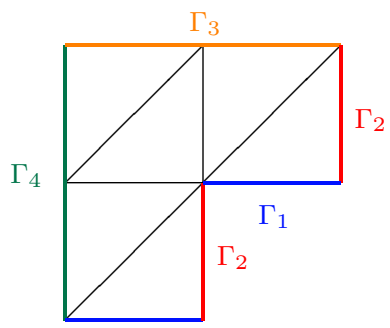

$\Gamma_{1}$

FIG. 1. Example 5.2.1: L-shaped domain.

5.2.1. Example 1. We use an example on the L-shaped domain, which is given by $\Omega=(-1,1)^{2} \backslash([0,1] \times(-1,0])$ with $\Gamma=\Gamma_{N}$; see Figure 1 . The control is defined on the whole boundary. The box constraints are given by $u^{a}=-0.5$ and $u^{b}=0.5$. The reaction term $\alpha$ and the regularization parameter $\omega$ are taken as $\alpha=1$ and $\omega=1$, respectively. The remaining data of the problem are defined in polar coordinates $(r, \theta)$ :

$$
\begin{gathered}
f(r, \theta)=0, \quad u^{d}(r, \theta)=0, \quad g^{N}(r, \theta)=-\operatorname{Proj}_{u^{a}, u^{b}}\left(r^{2 / 3} \sin \left(\frac{2}{3} \theta\right)\right), \\
y^{d}(r, \theta)=r^{2 / 3} \sin \left(\frac{2}{3} \theta\right), \\
r^{N}(r, \theta)=\left(\begin{array}{c}
\frac{2}{3} r^{-1 / 3} \cos (\theta) \sin \left(\frac{2}{3} \theta\right)-\frac{2}{3} r^{-1 / 3} \sin (\theta) \cos \left(\frac{2}{3} \theta\right) \\
\frac{2}{3} r^{-1 / 3} \sin (\theta) \sin \left(\frac{2}{3} \theta\right)+\frac{2}{3} r^{-1 / 3} \cos (\theta) \cos \left(\frac{2}{3} \theta\right)
\end{array}\right) \cdot \mathbf{n}_{\Gamma_{N}},
\end{gathered}
$$

where $r=\sqrt{x_{1}^{2}+x_{2}^{2}} \forall\left(x_{1}, x_{2}\right) \in \Omega$ and $\theta=\left\{\begin{array}{cc}\operatorname{atan} 2\left(x_{1}, x_{2}\right), & \operatorname{atan} 2\left(x_{1}, x_{2}\right) \geq 0, \\ \operatorname{atan} 2\left(x_{1}, x_{2}\right)+2 \pi, & \operatorname{atan} 2\left(x_{1}, x_{2}\right)<0\end{array}\right.$ with the function atan $2\left(x_{1}, x_{2}\right)$, i.e., the four-quadrant inverse tangent (arctangent) of $x_{1}$ and $x_{2}$. Note that the function atan2 $\left(x_{1}, x_{2}\right)$ is defined in MATLAB.

The analytical solutions of the state, adjoint, control, and cocontrol are given by

$$
\begin{aligned}
y(r, \theta) & =0, \\
p(r, \theta) & =r^{2 / 3} \sin \left(\frac{2}{3} \theta\right), \\
u(r, \theta) & =\operatorname{Proj}_{u^{a}, u^{b}}\left(r^{2 / 3} \sin \left(\frac{2}{3} \theta\right)\right), \\
\sigma(r, \theta) & =r^{2 / 3} \sin \left(\frac{2}{3} \theta\right)-\operatorname{Proj}_{u^{a}, u^{b}}\left(r^{2 / 3} \sin \left(\frac{2}{3} \theta\right)\right),
\end{aligned}
$$

respectively.

The adjoint exhibits a typical singularity at the reentrant corner of the domain $\Omega$; see Figure 2. Figure 3 displays the computed control $u$ and the computed cocontrol $\sigma$ on the Neumann boundary. We observe that the inactive set is equal to

$$
\mathcal{I}=[-0.78,0] \times\{-1\} \cup[0,1] \times\{0\} \cup\{0\} \times[-1,0] \cup\{1\} \times[0,0.78] .
$$

The initial mesh is generated by starting first by dividing $\Omega$ into uniform squares and then dividing each square into two triangles. It should be emphasized that we 


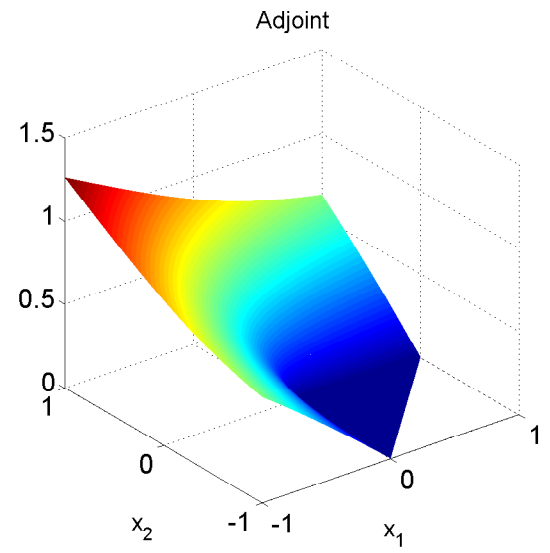

FIG. 2. Example 5.2.1: Computed solution of the adjoint p.

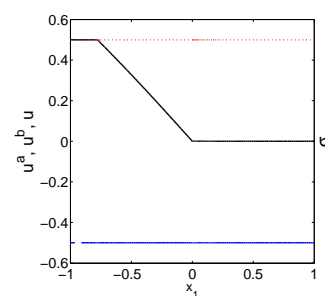

(a) $\Gamma_{1}=[-1,0] \times\{-1\} \cup[0,1] \times\{0\}$

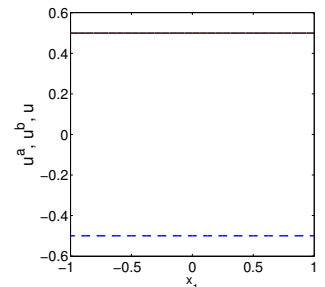

(c) $\Gamma_{3}=[-1,1] \times\{1\}$
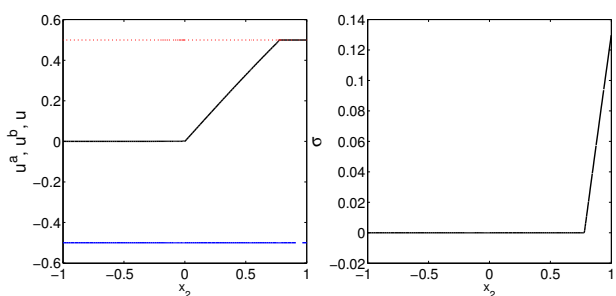

(b) $\Gamma_{2}=\{0\} \times[-1,0] \cup\{1\} \times[0,1]$
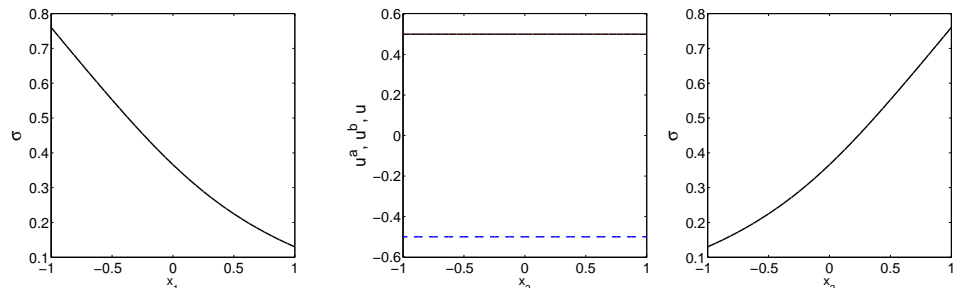

(d) $\Gamma_{4}=\{-1\} \times[-1,1]$

FIG. 3. Example 5.2.1: The computed control $u$ and the computed cocontrol $\sigma$ on the Neumann boundary regions.

are working with a single mesh for all variables. Consequently, the mesh reflects regions of substantial change in the variables. Figure 4 shows the adaptively generated triangulations after seven refinement steps with $\Theta=0.50$ in the bulk criterion. Most refinements occur around the reentrant corner, where the adjoint has the singularity, and on the boundary, where the inactive set $\mathcal{I}$ is defined, as we expected.

Figure 5 displays the performance of the error estimator proposed in section 4 in terms of the number of vertices for the marking parameter $\Theta=0.5$. The left plot shows the effectivity index of the estimator, which is the ratio between the error measured in the $\mid\|\cdot\|$-norm for the state and adjoint, and the $L^{2}$-norm for the control and cocontrol and the estimator, defined in (5.3). In order to obtain a constant ratio, a few more adaptive steps are still necessary. The middle plot displays that the error and estimator $\eta$ decay with a rate close to the optimal rate $N^{-1 / 2}$, where $N$ is the 


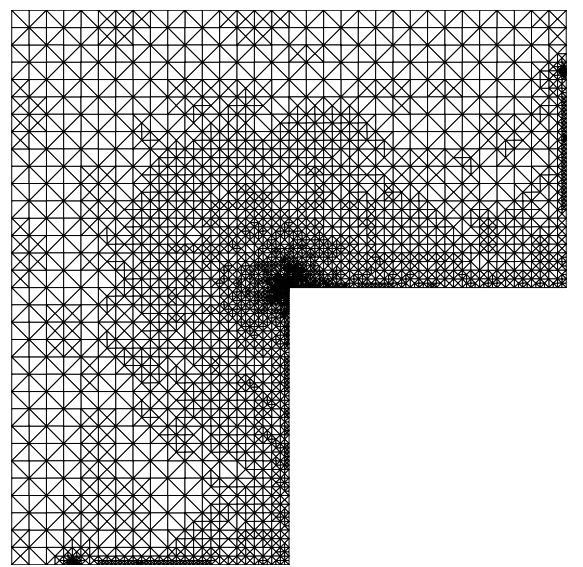

FIG. 4. Example 5.2.1: Adaptively generated mesh after 7 refinement steps with $\Theta=0.50$ in the bulk criterion.

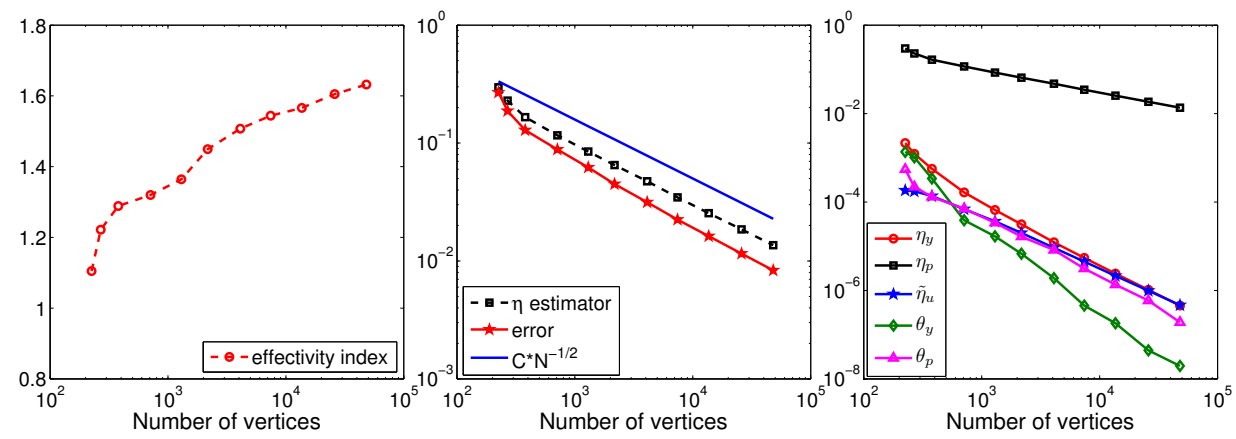

Fig. 5. Example 5.2.1: The left plot shows the effectivity index. The middle plot shows the decay of the total error and estimator. The right plot shows the components of the error estimator and data oscillation. The marking parameter is $\Theta=0.50$ in the bulk criterion.

number of vertices. Last, the right plot shows the actual sizes of the state, adjoint, and control related components of the error estimator and data oscillation. As can be expected, the adjoint component of the estimator is dominant due to the singularity at the reentrant corner of $\Omega$. Since the desired control $u^{d}$ and the bounds of the control, i.e., $u^{a}, u^{b}$, are constants, the data oscillation of the control $\theta_{u}$ is equal to zero.

We next have a closer look at the convergence of the state, adjoint, control, and cocontrol variables. Figure 6 illustrates the errors of the state and adjoint in the $\mid\|\cdot\|$ and $L^{2}$-norms, and of the control and cocontrol in the $L^{2}$-norm on adaptively and uniformly refined meshes with various marking parameters $\Theta=0.3,0.5,0.8$. For all cases, the adaptive refinements lead to better approximate solutions than the uniform refinements. Although the smaller $\Theta$ requires more refinement loops, it produces more accurate results due to the obtained optimal mesh.

5.2.2. Example 2. This example is taken from [36]. Kohls, Rösch, and Siebert have solved this example by using a hierarchical estimator, discretized by a continuous finite element approximation for the state and adjoint, and by a discontinuous finite element approximation for the control. We let $\Omega=[0,3]^{2}$ with $\Gamma=\Gamma_{N}$. However, the boundary control is only considered on $\{0\} \times[1,2]$. The reaction term $\alpha$ and the 

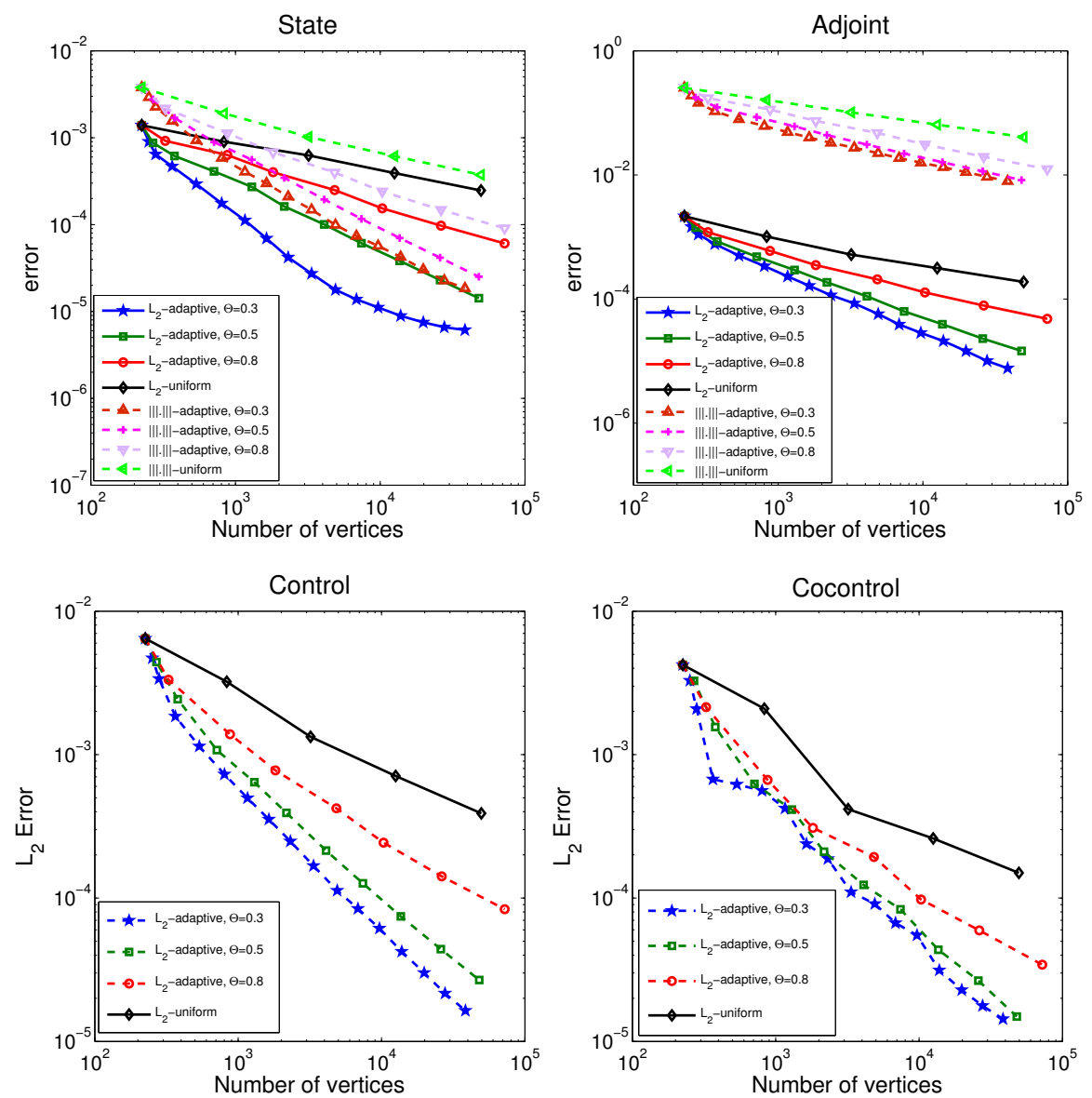

FIG. 6. Example 5.2.1: The global errors of the state, adjoint in $\|\cdot\| \mid$ and $L^{2}$-norms (top), and of the control and cocontrol in $L^{2}$-norm on adaptively and uniformly refined meshes with various marking parameters $\Theta=0.3,0.5,0.8$ in the bulk criterion.

regularization parameter $\omega$ are taken as $\alpha=1$ and $\omega=1$, respectively. The remaining setup of the problem is as follows:

$$
\begin{aligned}
& f\left(x_{1}, x_{2}\right)=e^{-10\left(x_{1}^{2}+x_{2}^{2}\right)}\left(41-400\left(x_{1}^{2}+x_{2}^{2}\right)\right), \\
& u^{d}\left(x_{1}, x_{2}\right)=0, \\
& g^{N}\left(x_{1}, x_{2}\right)= \\
& \begin{cases}-\operatorname{Proj}_{u^{a}, u^{b}}\left(\frac{Z}{2 n}\left((2 n+1)\left(\frac{2}{3} x_{2}-1\right)-\left(\frac{2}{3} x_{2}-1\right)^{2 n+1}\right)\right), & \left(x_{1}, x_{2}\right) \in\{0\} \times[1,2], \\
-60 e^{-10\left(9+y^{2}\right)}, & x_{1}=3, \\
-60 e^{-10\left(x^{2}+9\right),} & x_{2}=3, \\
0, & \text { otherwise, }\end{cases} \\
& y^{d}\left(x_{1}, x_{2}\right)= \\
& e^{-10\left(x_{1}^{2}+x_{2}^{2}\right)}+Z\left(\frac{2 n+1}{2 n}\left(\frac{2}{3} x_{2}-1\right)+\frac{8 n+4}{9}\left(\frac{2}{3} x_{2}-1\right)^{2 n-1}-\left(\frac{2}{3} x_{2}-1\right)^{2 n+1}\right), \\
& r^{N}\left(x_{1}, x_{2}\right)=0 .
\end{aligned}
$$



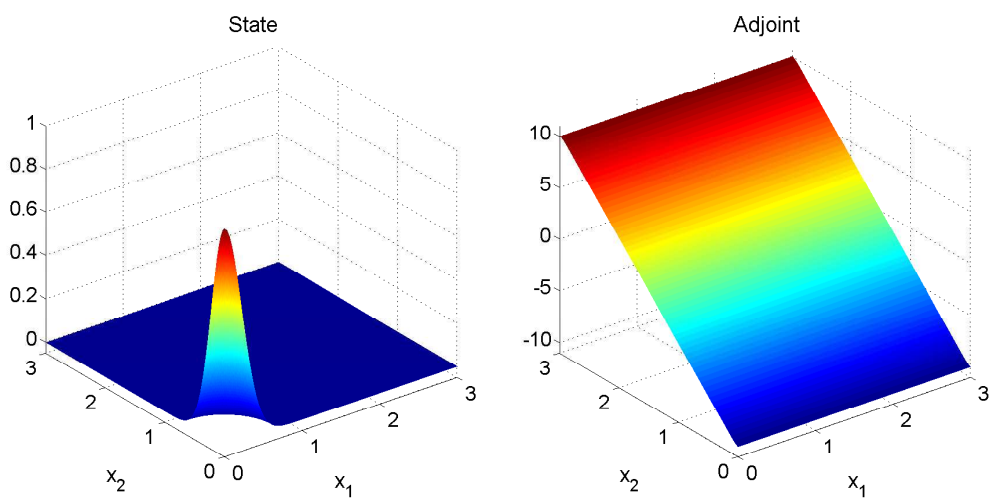

FiG. 7. Example 5.2.2: Computed solutions of the state $y$ (left) and the adjoint (right).

The analytical solutions of the state, adjoint, control, and cocontrol are given by

$$
\begin{aligned}
y\left(x_{1}, x_{2}\right)= & e^{-10\left(x_{1}^{2}+x_{2}^{2}\right)}, \\
p\left(x_{1}, x_{2}\right)= & \frac{Z}{2 n}\left((2 n+1)\left(\frac{2}{3} x_{2}-1\right)-\left(\frac{2}{3} x_{2}-1\right)^{2 n+1}\right), \\
u\left(x_{1}, x_{2}\right)= & \operatorname{Proj}_{u^{a}, u^{b}}\left(\frac{Z}{2 n}\left((2 n+1)\left(\frac{2}{3} x_{2}-1\right)-\left(\frac{2}{3} x_{2}-1\right)^{2 n+1}\right)\right), \\
\sigma\left(x_{1}, x_{2}\right)= & \frac{Z}{2 n}\left((2 n+1)\left(\frac{2}{3} x_{2}-1\right)-\left(\frac{2}{3} x_{2}-1\right)^{2 n+1}\right) \\
& -\operatorname{Proj}_{u^{a}, u^{b}}\left(\frac{Z}{2 n}\left((2 n+1)\left(\frac{2}{3} x_{2}-1\right)-\left(\frac{2}{3} x_{2}-1\right)^{2 n+1}\right)\right),
\end{aligned}
$$

respectively, with $Z=10, n=20$. The components of the error estimator exhibit local refinements in different regions of the domain due to the particular features of the state, the adjoint, and the control. The state $y$ needs more refinement around the origin due to the shape of the narrow exponential peak. The adjoint $p$ displays a boundary layer close to $x_{2}=0$ and $x_{2}=3$. These features can be observed in Figure 7 .

To observe the sensitivity of the adaptive algorithm with respect to the changes of the active and inactive sets, we test the example with different box constraints:

Inactive case: We first consider the control constraints as

$$
u^{a}=-5 \text { and } u^{b}=5 .
$$

Figure 8 reveals the adaptively refined mesh (left) and the computed control (right) for the inactive case. We observe that the control is between the lower bound and the upper bound, i.e., $u^{a}<u<u^{b}$. Therefore, the inactive set $\mathcal{I}$ is equal to all of the control boundary, i.e., $\mathcal{I}=\{0\} \times[1,2]$. In the adaptive refinement, our error indicator $\widetilde{\eta}_{u}$ (4.46) catches the inactive set well (see Figure 8 (left)), after 13 adaptive refinement steps with $\Theta=0.5$ in the bulk criterion. Further, the resolution of the state and the adjoint occur as expected.

Mixed case I: The control constraints are now considered as

$$
u^{a}=-2 \quad \text { and } \quad u^{b}=0 .
$$



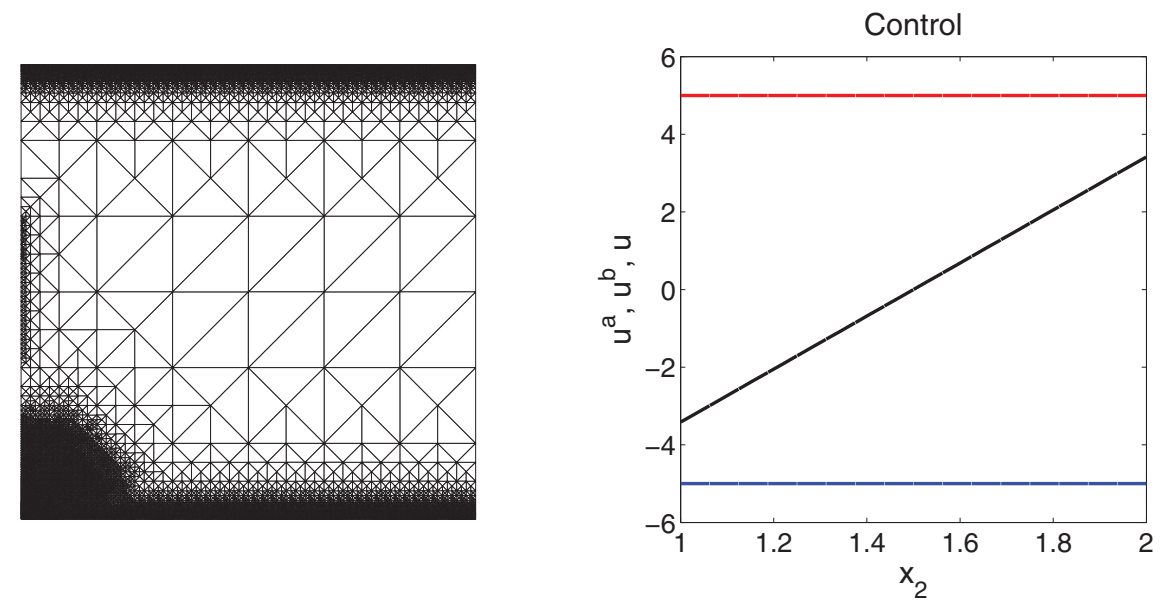

FIG. 8. Example 5.2.2: Adaptively generated mesh (left) after 13 adaptive refinement steps with $\Theta=0.5$, and the computed control $u$ (right) for the inactive case.
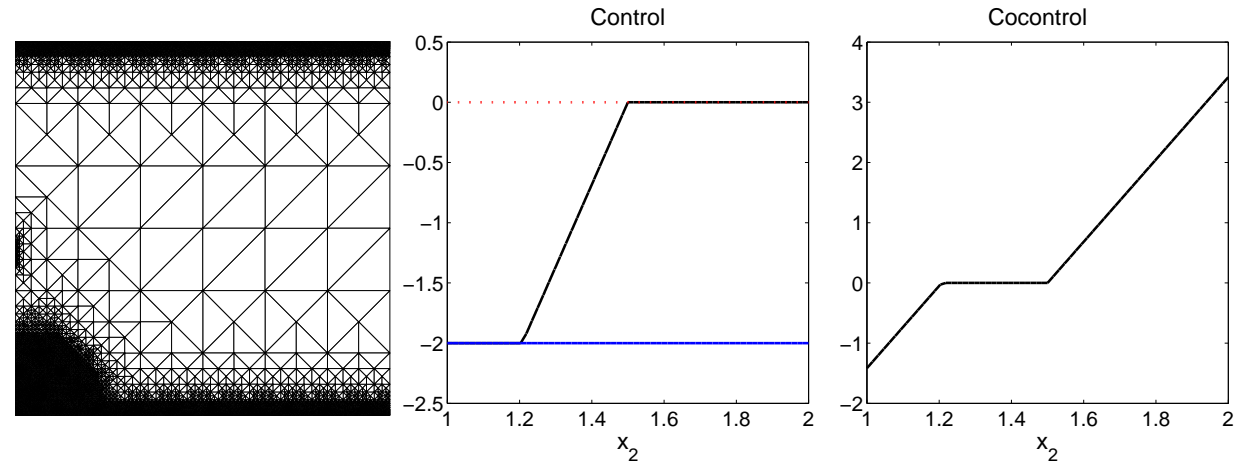

FIG. 9. Example 5.2.2: Adaptively generated mesh (left) after 13 adaptive refinement steps with $\Theta=0.5$, and the computed control $u$ (middle) and cocontrol $\sigma$ (right) for the mixed case I.

Now, the value of the control varies between the lower bound and the upper bound:

$$
u=u^{a} \quad \text { for } x_{2} \in[1,1.2] \text { and } u=u^{b} \quad \text { for } x_{2} \in[1.4,2] .
$$

Therefore, the inactive set is $\mathcal{I}=\{0\} \times[1.2,1.4]$. Figure 9 reveals that the inactive set of the mixed case I is picked out well in the adaptive refinement.

Mixed case II: We finally consider the control constraints as

$$
u^{a}=0 \quad \text { and } \quad u^{b}=2 .
$$

Now, the value of the control varies between the lower bound and the upper bound as in the previous case:

$$
u=u^{a} \quad \text { for } x_{2} \in[1,1.5] \text { and } \quad u=u^{b} \quad \text { for } x_{2} \in[1.8,2] .
$$

Therefore, the inactive set is $\mathcal{I}=\{0\} \times[1.5,1.8]$. As in the previous cases, the inactive set of the mixed case II is picked out well in the adaptive refinement; see Figure 10 (left).

We next have a closer look at some properties of the proposed estimator for the inactive case. Figure 11 (left) displays the ratio between the error and the estimator, called the effectivity index for the inactive case with the marking parameter $\Theta=0.50$. 

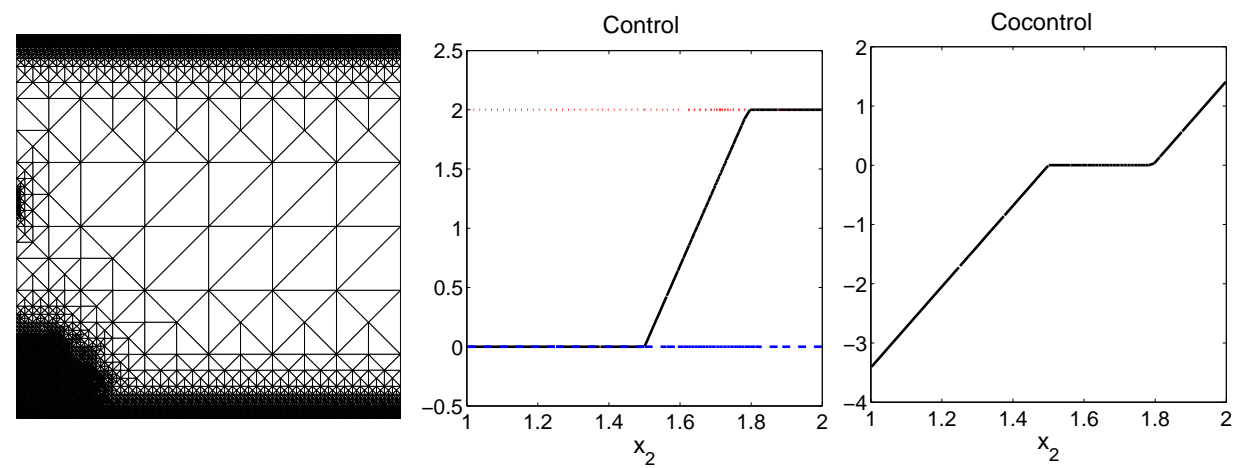

FIG. 10. Example 5.2.2: Adaptively generated mesh (left) after 13 adaptive refinement steps with $\Theta=0.5$, and the computed control $u$ (middle) and cocontrol $\sigma$ (right) for the mixed case II.
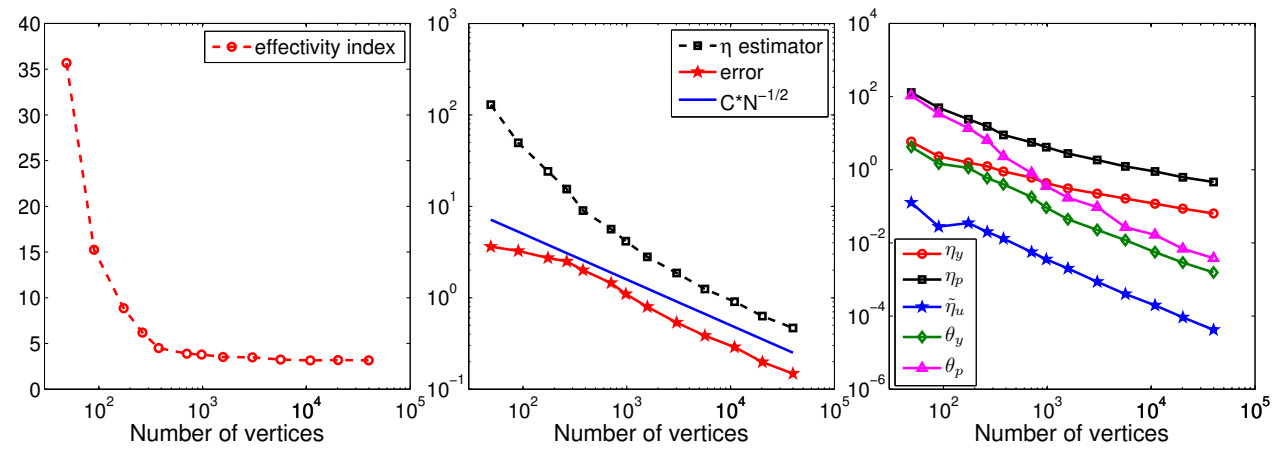

FIG. 11. Example 5.2.2: The left plot shows the effectivity index. The middle plot shows the decay of the total error and estimator. The right plot shows the components of the error estimator and the data oscillation. The results are obtained for the inactive case with $\Theta=0.50$ in the bulk criterion.

The ratio converges to a constant after a few iterations. The middle plot in Figure 11 shows the decay of the error and estimator versus the number of vertices for the adaptive refinement. The estimator underestimates the error by an almost constant factor. We observe that the behavior of the error and the estimator is similar to the results obtained in [36]. The right plot in Figure 11 shows the actual size of the state, adjoint, and control related components of the error estimator and the data oscillations for the inactive case. The refinement process is dominated by the contribution of the adjoint.

Figure 12 illustrates the errors of the state and adjoint in the $\|\cdot\| \mid$ and $L^{2}$-norms, and of the control in the $L^{2}$-norm on adaptively and uniformly refined meshes with various marking parameters $\Theta=0.3,0.5,0.8$. The adaptive refinements lead to better approximate solutions than uniform refinements.

Last, we make a comparison of the control estimators $\eta_{u}, \widetilde{\eta}_{u}$ for the mixed cases I and II. The left sides of Figures 13 and 14 show the meshes generated by $\eta_{u}$. As we mentioned in Remark 4.10, the estimator $\eta_{u}$ contributed from the approximation error of the control does not lead to a localization of refinement of the inactive set; cf. Figures 13 and 14. Also, the convergence of the estimator $\eta_{u}$ is worse than the control estimator $\widetilde{\eta}_{u}$; see Figures 13 and 14 (right). Therefore, in our numerical computations, we use $\widetilde{\eta}_{u}$ as a control estimator. This numerical computation is also mentioned in [36, Remark 6.1]. 

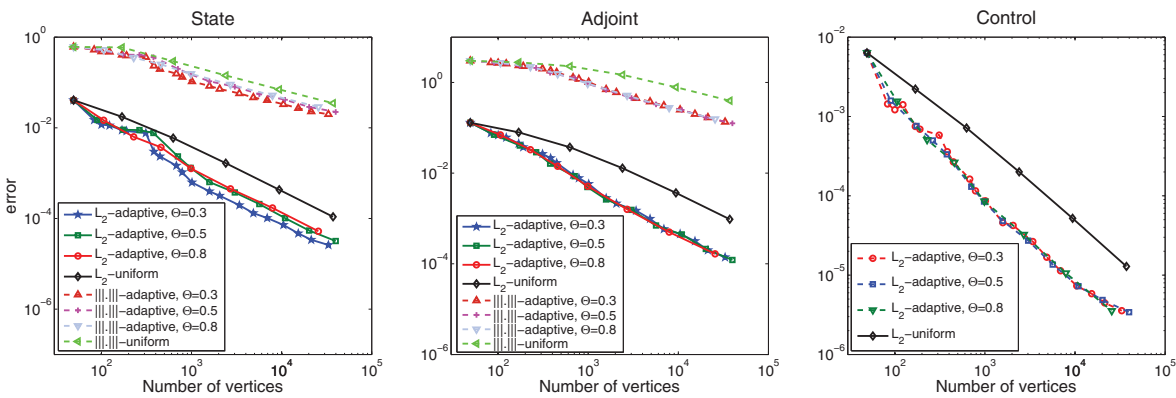

FIG. 12. Example 5.2.2: Errors of the state $y$ (left) and adjoint $p$ (middle) in $\|\cdot\| \mid$ and $L^{2}$ norms, and the control $u$ (right) in $L^{2}$-norm on adaptively and uniformly refined meshes for the inactive case with various marking parameters $\Theta=0.3,0.5,0.8$ in the bulk criterion.
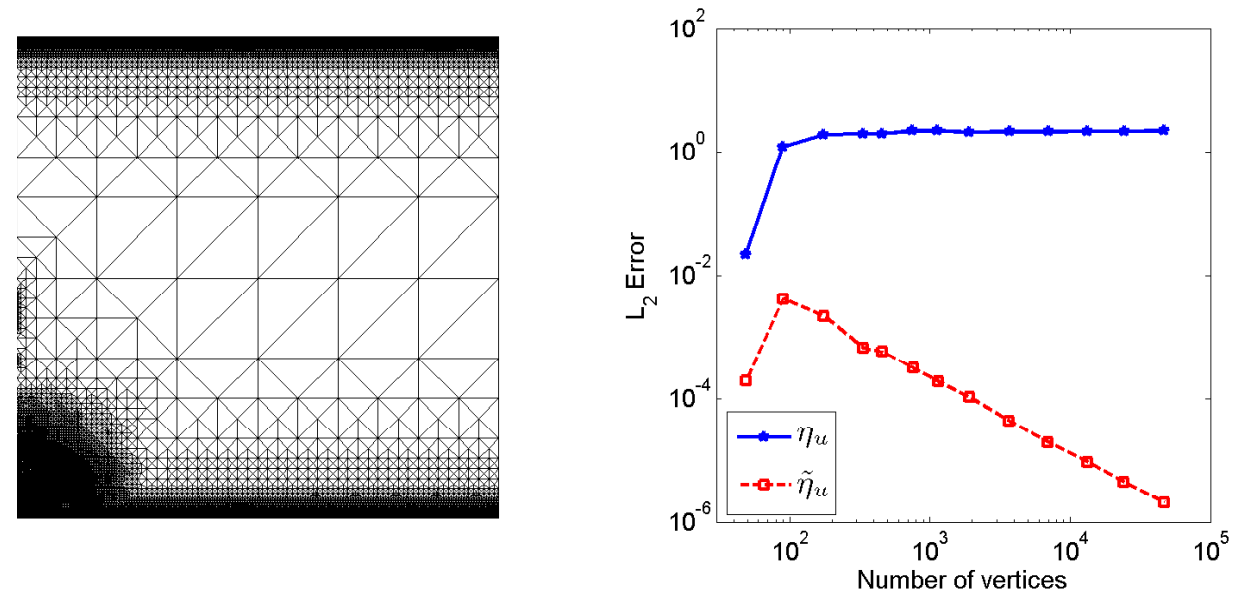

FIG. 13. Example 5.2.2: Adaptively generated mesh (left) by using $\eta_{u}$ after 13 adaptive refinement steps with $\Theta=0.5$, and convergence of the error estimators $\eta_{u}$ and $\tilde{\eta}_{u}$ (right) for the mixed case I.

5.2.3. Example 3. We use the example considered in [19]. Gaevskaya et al. have solved this example by using a residual-type error estimator, discretized by a continuous finite element approximation. In this example, we use nonconstant lower and upper bounds for the control, which are highly oscillating constraints. The data of the problem are

$$
\begin{aligned}
\Omega & =(0,1)^{2}, \quad \Gamma_{N}=(0,1) \times\{0\}, \quad \Gamma_{D}=\partial \backslash \Gamma_{N}, \quad \alpha\left(x_{1}, x_{2}\right)=1, \\
\omega & =10^{-3}, \quad u^{d}\left(x_{1}, x_{2}\right)=0, \\
y^{d}\left(x_{1}, x_{2}\right) & = \begin{cases}0, & x_{1} \leq 0.5, \\
1, & 0.5<x_{1}<0.75, \\
-1, & 0.75<x_{1},\end{cases} \\
f\left(x_{1}, x_{2}\right) & =0, \quad g^{D}\left(x_{1}, x_{2}\right)=0, \quad g^{N}\left(x_{1}, x_{2}\right)=0, \\
h^{N}\left(x_{1}, x_{2}\right) & =0, \quad u^{a}=\sin \left(8 \pi x_{1}\right), \quad u^{b}=2+\cos \left(\pi / 2+8 x_{1}\right) .
\end{aligned}
$$

Figures 15 and 16 show the computed solutions of the state $y$, the adjoint $p$, the control $u$, and the cocontrol $\sigma$, respectively. The control switches from the lower 

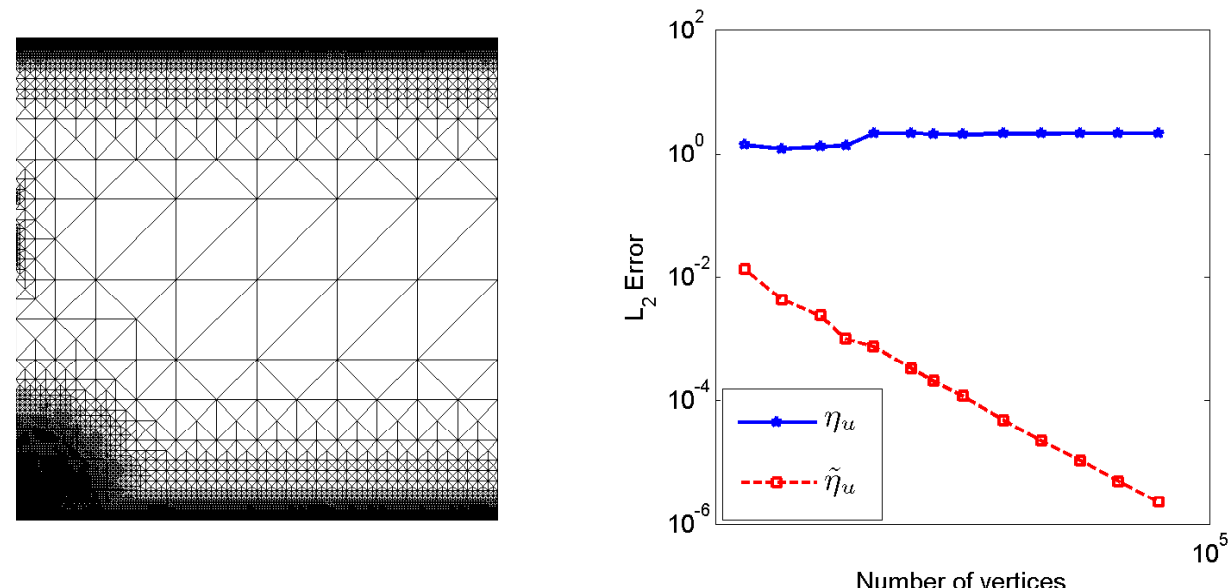

FIG. 14. Example 5.2.2: Adaptively generated mesh (left) by using $\eta_{u}$ after 13 adaptive refinement steps with $\Theta=0.5$, and convergence of the error estimators $\eta_{u}$ and $\tilde{\eta}_{u}$ (right) for the mixed case II.
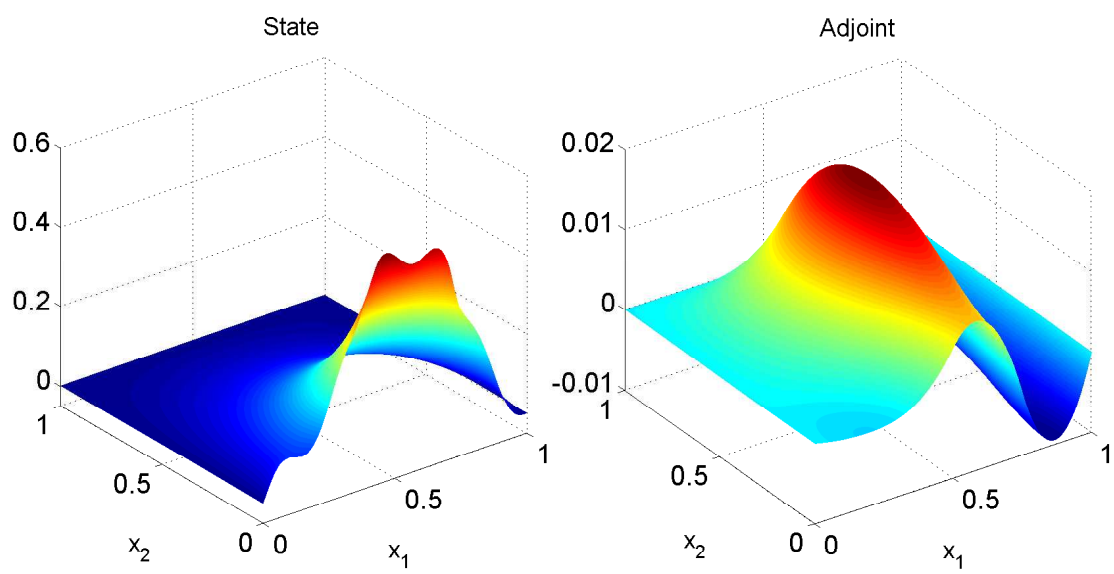

FiG. 15. Example 5.2.3: Computed solutions of the state $y$ (left) and of the adjoint p (right).

to the upper bound and back again to the lower bound on $\Gamma_{N}$. This is an almost "bang-bang"-type optimal control.

The initial mesh is generated by starting by first dividing $\Omega$ into $8 \times 8$ uniform squares and then dividing each square into two triangles as in the previous examples. Adaptively generated meshes after six (left) and eight (right) refinements are displayed in Figure 17 with $\Theta=0.45$ in the bulk criterion. More refinements occur on the boundary, where the inactive set $\mathcal{I}$ is defined and on the right side of the mesh, i.e., $(0.5,1) \times(0,1)$.

The components of the residual-type a posteriori error estimator and data oscillations are presented in Table 1 on the mesh hierarchy with $\Theta=0.45$ in the bulk criterion. We observe that the dominating contributions such as the state estimator $\eta_{y}$, the adjoint estimator $\eta_{p}$, and the control oscillation $\theta_{u}$ are smaller than those obtained in [19] for approximately the same number of vertices. 

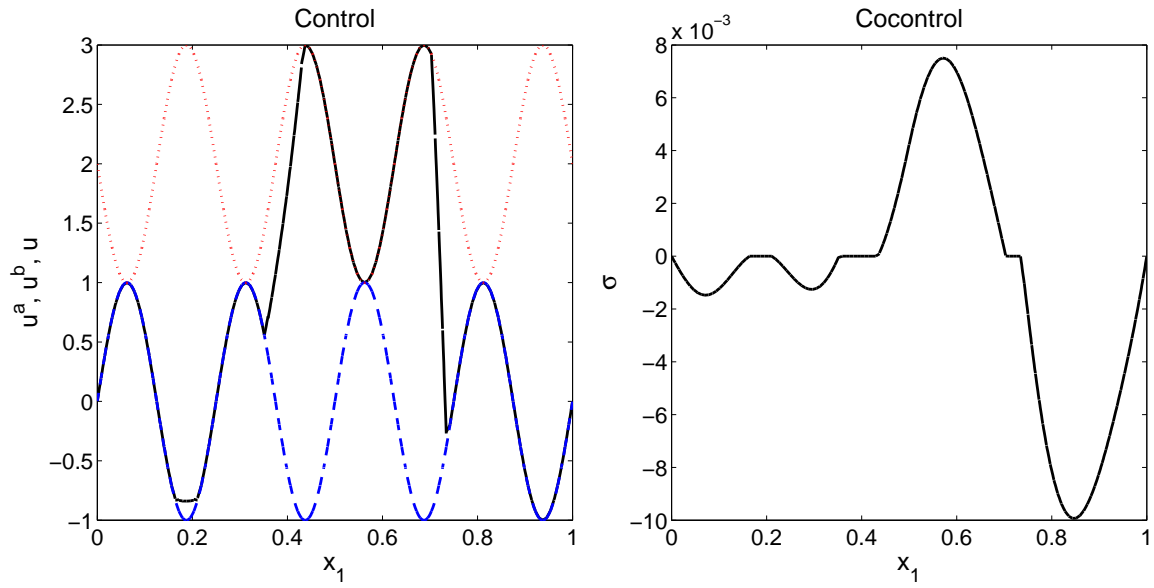

Fig. 16. Example 5.2.3: Computed solutions of the control u (left) and of the cocontrol $\sigma$ (right). The lower and upper bounds on the control, i.e., $u^{a}$ and $u^{b}$, are shown as "dashed (blue)" and "dotted (red)" lines, respectively.
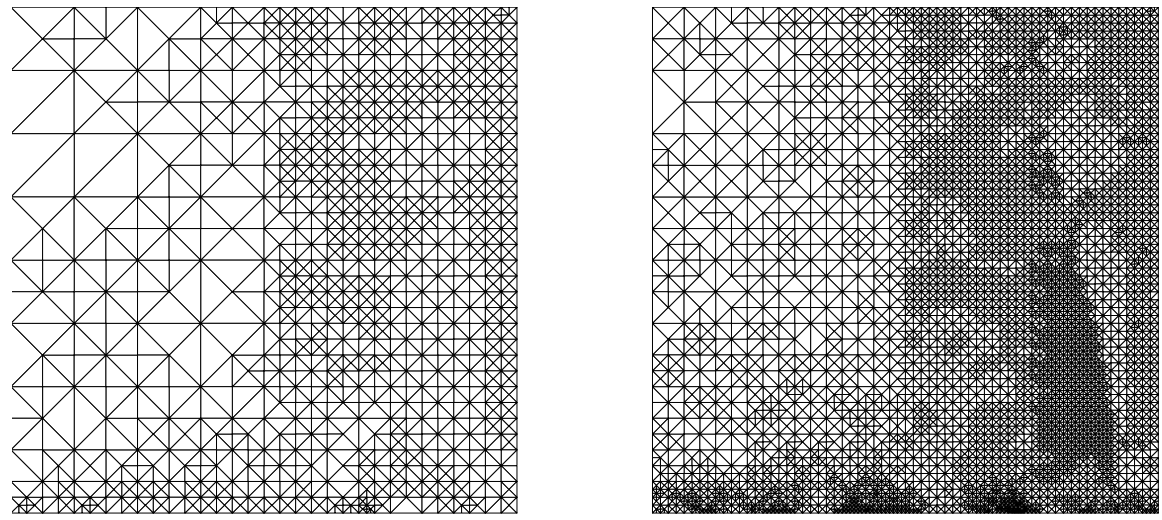

FIG. 17. Example 5.2.3: Adaptively generated meshes after 6 (left) and 8 (right) refinement steps with $\Theta=0.45$ in the bulk criterion.

TABLE 1

Example 5.2.3: Components of the error estimator and data oscillation for $\Theta=0.45$.

\begin{tabular}{c|c|c|c|c|c}
\hline \# vertices & $\eta_{y}$ & $\eta_{p}$ & $\widetilde{\eta}_{u}$ & $\theta_{p}$ & $\theta_{u}$ \\
\hline 81 & $4.83 \mathrm{e}-01$ & $1.20 \mathrm{e}-01$ & $5.30 \mathrm{e}-06$ & $8.07 \mathrm{e}-02$ & $1.00 \mathrm{e}+00$ \\
167 & $3.70 \mathrm{e}-01$ & $8.03 \mathrm{e}-02$ & $2.84 \mathrm{e}-06$ & $3.93 \mathrm{e}-02$ & $6.35 \mathrm{e}-01$ \\
332 & $2.80 \mathrm{e}-01$ & $5.81 \mathrm{e}-02$ & $9.21 \mathrm{e}-07$ & $2.37 \mathrm{e}-02$ & $3.89 \mathrm{e}-01$ \\
582 & $2.49 \mathrm{e}-01$ & $4.42 \mathrm{e}-02$ & $5.52 \mathrm{e}-07$ & $1.39 \mathrm{e}-02$ & $1.37 \mathrm{e}-01$ \\
1174 & $1.83 \mathrm{e}-01$ & $3.26 \mathrm{e}-02$ & $2.60 \mathrm{e}-07$ & $9.51 \mathrm{e}-03$ & $7.30 \mathrm{e}-02$ \\
2116 & $1.32 \mathrm{e}-01$ & $2.46 \mathrm{e}-02$ & $1.23 \mathrm{e}-07$ & $6.02 \mathrm{e}-03$ & $3.91 \mathrm{e}-02$ \\
4055 & $9.57 \mathrm{e}-02$ & $1.90 \mathrm{e}-02$ & $5.96 \mathrm{e}-08$ & $3.80 \mathrm{e}-03$ & $1.75 \mathrm{e}-02$ \\
7234 & $6.92 \mathrm{e}-02$ & $1.44 \mathrm{e}-02$ & $2.97 \mathrm{e}-08$ & $2.11 \mathrm{e}-03$ & $9.76 \mathrm{e}-03$ \\
\hline
\end{tabular}

6. Conclusions. In this paper, we have studied a posteriori error analysis of the SIPG method for boundary optimal control problems governed by elliptic PDEs with bilateral control constraints. Piecewise linear polynomials are used to discretize the unknown variables. Lower and upper error estimates are derived to show the 
efficiency and reliability of the proposed error estimator by invoking data oscillations. The numerical results show that the adaptive refinements are superior to uniform refinements. Future work will include the extension of our results to Dirichlet boundary optimal control problems and convection-diffusion problems.

Acknowledgments. We would like to thank Martin Stoll for helpful discussions about this research. The authors also would like to express their sincere thanks to the referees for their most valuable suggestions.

\section{REFERENCES}

[1] R. A. Adams, Sobolev Spaces, Academic Press, Orlando, FL, 1975.

[2] M. Ainsworth, A posteriori error estimation for discontinuous Galerkin finite element approximation, SIAM J. Numer. Anal., 45 (2007), pp. 1777-1798.

[3] D. N. Arnold, F. Brezzi, B. Cockburn, and L. D. Marini, Unified analysis of discontinuous Galerkin methods for elliptic problems, SIAM J. Numer. Anal., 39 (2002), pp. 1749-1779.

[4] I. BABUŠKa AND W. C. RheinBoldt, Error estimates for adaptive finite element computations, SIAM J. Numer. Anal., 15 (1978), pp. 736-754.

[5] G. A. Baker, W. N. Jureidini, and O. A. Karakashian, Piecewise solenoidal vector fields and the Stokes problem, SIAM J. Numer. Anal., 27 (1990), pp. 1466-1485.

[6] R. Becker, H. Kapp, And R. Rannacher, Adaptive finite element methods for optimal control of partial differential equations: Basic concept, SIAM J. Control Optim., 39 (2000), pp. 113-132.

[7] R. BECKer And S. P. MaO, Quasi-optimality of an adaptive finite element method for an optimal control problem, Comput. Methods Appl. Math., 11 (2011), pp. 107-128.

[8] O. Benedix AND B. Vexler, A posteriori error estimation and adaptivity for elliptic optimal control problems with state constraints, Comput. Optim. Appl., 44 (2009), pp. 3-25.

[9] M. Bergounioux, K. Ito, and K. Kunisch, Primal-dual strategy for constrained optimal control problems, SIAM J. Control Optim., 37 (1999), pp. 1176-1194.

[10] S. C. Brenner and L. R. Scott, The Mathematical Theory of Finite Element Methods, 2nd ed., Springer, Berlin, 2002.

[11] A. Cangiani, J. Chapman, E. H. Georgoulis, and M. Jensen, On local super-penalization of interior penalty discontinuous Galerkin methods, Int. J. Numer. Anal. Model., 11 (2014), pp. 478-495.

[12] E. CASAS AND V. Dhamo, Error estimates for the numerical approximation of Neumann control problems governed by a class of quasilinear elliptic equations, Comput. Optim. Appl., 52 (2012), pp. 719-756.

[13] L. Chen, iFEM: An Innovative Finite Element Methods Package in MATLAB, Technical report, Department of Mathematics, University of California, Irvine, CA, 2008.

[14] D. Clever, J. Lang, S. Ulbrich, ANd J. C. ZIems, Combination of an adaptive multilevel SQP method and a space-time adaptive PDAE solver for optimal control problems, Procedia Comput. Sci., 1 (2010), pp. 1435-1443.

[15] L. Dedì, S. Micheletti, And S. Perotto, Anisotropic error control for environmental applications, Appl. Numer. Math., 58 (2008), pp. 1320-1339.

[16] W. Dörfler, A convergent adaptive algorithm for Poisson's equation, SIAM J. Numer. Anal., 33 (1996), pp. 1106-1124.

[17] H. O. FATtoRInI, Infinite Dimensional Optimization and Optimal Control, Encyclopedia Math. Appl. 62, Cambridge University Press, Cambridge, New York, 1999.

[18] H. J. S. Fernando, S. M. Lee, and J. Anderson, Urban fluid mechanics: Air circulation and contaminant dispersion in cities, Environ. Fluid Mech., 1 (2001), pp. 107-164.

[19] A. Gaevskaya, R. H. W. Hoppe, Y. Iliash, and M. Kieweg, A posteriori error analysis of control constrained distributed and boundary control problems, in Proc. Conf. Scientific Computing. Russian Academy of Sciences, Moscow, 2006.

[20] A. Gaevskaya, Y. Iliash, M. Kieweg, and R. H. W. Hoppe, Convergence analysis of an adaptive finite element method for distributed control problems with control constraints, Control of Coupled Partial Differential Equations, Birkhäuser, Basel, 2007, pp. 47-68.

[21] W. Gong AND N. Yan, Adaptive finite element method for elliptic optimal control problems: Convergence and optimality, Numer. Math., 135 (2017), pp. 1121-1170.

[22] R. Griesse And S. Volkwein, A primal-dual active set strategy for optimal boundary control of a nonlinear reaction-diffusion system, SIAM J. Control Optim., 44 (2005), pp. 467-494.

Copyright $@$ by SIAM. Unauthorized reproduction of this article is prohibited. 
[23] A. Günther and M. Hinze, A posteriori error control of a state constrained elliptic control problem, J. Numer. Math., 16 (2008), pp. 307-322.

[24] J. S. Hesthaven and T. Warburton, Nodal Discontinuous Galerkin Methods: Analysis, Algorithms, and Applications, Springer, Berlin, 2008.

[25] M. Hintermüller AND R. H. W. Hoppe, Goal-oriented adaptivity in pointwise state constrained optimal control of partial differential equations, SIAM J. Control Optim., 48 (2010), pp. 5468-5487.

[26] M. Hintermüller, R. H. W. Hoppe, Y. Iliash, and M. Kieweg, An a posteriori error analysis of adaptive finite element methods for distributed elliptic control problems with control constraints, ESAIM Control Optim. Calc. Var., 14 (2008), pp. 540-560.

[27] M. Hintermüller, K. Ito, And K. Kunisch, The primal-dual active set strategy as a semismooth Newton method, SIAM J. Optim., 13 (2002), pp. 865-888.

[28] M. Hinze and M. Matthes, A note on variational discretization of elliptic Neumann boundary control, Control Cybernet., 38 (2009), pp. 577-591.

[29] M. Hinze, N. Yan, AND Z. Zhou, Variational discretization for optimal control governed by convection dominated diffusion equations, J. Comput. Math., 27 (2009), pp. 237-253.

[30] R. H. W. Hoppe, Y. Iliash, C. Iyyunni, and N. H. Sweilam, A posteriori error estimates for adaptive finite element discretizations of boundary control problems, J. Numer. Math., 14 (2006), pp. 57-82.

[31] R. H. W. Hoppe, G. Kanschat, and T. Warburton, Convergence analysis of an adaptive interior penalty discontinuous Galerkin method, SIAM J. Numer. Anal., 47 (2009), pp. 534550 .

[32] R. H. W. Hoppe And M. KIEWEG, Adaptive finite element methods for mixed control-state constrained optimal control problems for elliptic boundary value problems, Comput. Optim. Appl., 46 (2010), pp. 511-533.

[33] G. Kanschat, Discontinuous Galerkin Methods for Viscous Incompressible Flow, Adv. Numer. Math., Teubner, Wiesbaden, Germany, 2007.

[34] O. A. Karakashian and F. Pascal, A posteriori error estimates for a discontinuous Galerkin approximation of second-order elliptic problems, SIAM J. Numer. Anal., 41 (2003), pp. 2374-2399.

[35] O. A. Karakashian and F. Pascal, Convergence of adaptive discontinuous Galerkin approximations of second-order elliptic problems, SIAM J. Numer. Anal., 45 (2007), pp. 641-665.

[36] K. Kohls, A. Rösch, AND K. G. Siebert, A posteriori error analysis of optimal control problems with control constraints, SIAM J. Control. Optim., 52 (2014), pp. 1832-1861.

[37] K. Kohls, K. G. Siebert, AND A. Rösch, Convergence of adaptive finite elements for optimal control problems with control constraints, Trends in PDE Constrained Optimization, Springer, Cham, Switzerland, 2014, pp. 403-419.

[38] D. Leykekhman and M. Heinkenschloss, Local error analysis of discontinuous Galerkin methods for advection-dominated elliptic linear-quadratic optimal control problems, SIAM J. Numer. Anal., 50 (2012), pp. 2012-2038.

[39] R. LI, W. LIU, H. MA, AND T. TANG, Adaptive finite element approximation for distributed elliptic optimal control problems, SIAM J. Control Optim., 41 (2002), pp. 1321-1349.

[40] J.-L. Lions, Optimal Control of Systems Governed by Partial Differential Equations, Springer, Berlin, 1971.

[41] W. Liu AND N. YAn, A posteriori error estimators for a class of variational inequalities, J. Sci. Comput., 35 (2000), pp. 361-393.

[42] W. LiU AND N. YAN, A posteriori error estimates for convex boundary control problems, SIAM J. Numer. Anal., 39 (2001), pp. 73-99.

[43] W. Liu AND N. YAN, A posteriori error estimates for distributed convex optimal control problems, Adv. Comput. Math., 15 (2001), pp. 285-309.

[44] W. LiU AND N. YAN, Local a posteriori error estimates for convex boundary control problems, SIAM J. Numer. Anal., 47 (2009), pp. 1886-1908.

[45] B. Mohammadi and O. Pironneau, Applied Shape Optimization for Fluids, Oxford University Press, Oxford, 2001.

[46] P. Morin, R. H. Nochetto, And K. G. Siebert, Data oscillation and convergence of adaptive FEM, SIAM J. Numer. Anal., 38 (2000), pp. 466-488.

[47] D. Parra-Guevara and Y. N. Skiba, On optimal solution of an inverse air pollution problem: Theory and numerical approach, Math. Comput. Model., 43 (2006), pp. 766-778.

[48] B. RIvière, Discontinuous Galerkin methods for solving elliptic and parabolic equations, Theory and implementation, Frontiers Appl. Math., SIAM, Philadelphia, 2008.

[49] R. Verfürth, A Review of A Posteriori Error Estimation and Adaptive Mesh-Refinement Techniques, Adv. Numer. Math., Wiley Teubner, Chicester, 1996.

Copyright $@$ by SIAM. Unauthorized reproduction of this article is prohibited. 
[50] W. Wollner, A posteriori error estimates for a finite element discretization of interior point methods for an elliptic optimization problem with state constraints, Comput. Optim. App., 47 (2010), pp. 133-159.

[51] N. YAN AND Z. ZHOU, A priori and a posteriori error analysis of edge stabilization Galerkin method for the optimal control problem governed by convection-dominated diffusion equation, J. Comput. Appl. Math., 223 (2009), pp. 198-217.

[52] H. YÜCEl AND P. Benner, Adaptive discontinuous Galerkin methods for state constrained optimal control problems governed by convection diffusion equations, Comput. Optim. Appl., 62 (2015), pp. 291-321.

[53] H. YüCel, M. Heinkenschloss, and B. Karasözen, Distributed optimal control of diffusionconvection-reaction equations using discontinuous Galerkin methods, in Numer. Math. Adv. Appl., 2011, Springer, Berlon, 2013, pp. 389-397.

[54] H. YÜCEL AND B. KARASÖZEN, Adaptive symmetric interior penalty Galerkin (SIPG) method for optimal control of convection diffusion equations with control constraints, Optimization, 63 (2014), pp. 145-166.

[55] Z. ZHOU, X. YU, AND N. YAN, The local discontinuous Galerkin approximation of convectiondominated diffusion optimal control problems with control constraints, Numer. Methods Partial Differential Equations, 30 (2014), pp. 339-360.

Copyright $@$ by SIAM. Unauthorized reproduction of this article is prohibited. 